\title{
When ordinary becomes extraordinary: A postschool follow up study of the integrated vocational functioning of 50 workers with significant intellectual disabilities, 1984-2020
}

Lou Brown, Sharon Schmid*, Sarah Cutler*, Kim Kessler and Betsy Shiraga

University of Wisconsin and Community Work Services, Inc. Madison, WI, USA

Received 18 September 2020

Accepted 1 December 2020

\begin{abstract}
.
OBJECTIVE: The primary purpose is to share information about 50 individuals with significant intellectual disabilities, the lowest intellectually functioning 1\% of those who exited schools, from 1981 to 2003.

RESULTS: The 47 who attended the Madison Metropolitan School District (MMSD) received instruction in integrated nonschool work and related environments as part of their school experiences. After exiting school, the 50 functioned in integrated work settings for over 36 years. Information pertaining to the work environments in which they functioned, the work tasks performed, the reasons for changes in environments and tasks, the hours worked per week, the wages earned, the travel modes used, the lunch supports provided, the extra supports provided by Job Coaches, involvement in Community and Recreation activities and where each resided is presented. Social relationships with coworkers and others without disabilities, the mortality of the 50 workers and their parents and guardianship are also addressed.

CONCLUSION: The authors are not aware of any other postschool follow up study of so many workers with significant intellectual disabilities who functioned in integrated work settings for such long periods of time.
\end{abstract}

Keywords: Significant intellectual disabilities, integrated work, school to work transition, longitudinal post school follow up

\section{Introduction}

In the early 1970's the MMSD adopted a "Zero Exclusion" policy which opened its doors to all children with significant intellectual disabilities. Almost all were served in a segregated school. As time passed some of those directly involved contemplated the nature and meaning of the educational and related

\footnotetext{
*Address for correspondence: Sarah Cutler and Sharon Schmid, Community Work Services, Inc. 1402 Greenway Cross \#200 Madison, WI 53713, USA. Tel.:+ 1608233 0115; Fax: +1 608233 6886; E-mail: Sarahandsharon@cwsmadison.com.
}

services they were providing. Are we doing the right things? What happens when school ends? Can we help our students live better lives than those who preceded them? (Brown, Nietupski \& Nietupski, 1976). These and related questions led to the gathering of information about the postschool realities being experienced by adults of similar kinds and degrees of disabilities in Madison. The postschool lives of such persons consisted of staying in residences all day, being confined to a segregated workshop or activity centers, being on a waiting list for services and interacting only with family members, others with 
disabilities and persons paid to be with them. A few judged these segregated, nonproductive, isolated and otherwise limiting school outcomes unacceptable. They dreamed of postschool lives that were richer, more varied, safer, more interesting, challenging, respected and otherwise "more ordinary." Tangibly, more ordinary meant increasing: the number of environments in which the students would function in postschool life; the quantity and complexity of respected chronological age appropriate and functional skills they were given opportunities to learn and perform; the number of persons with and without disabilities with whom they interacted and developed social relationships; and the meaningful choices they could make about where they went, what they did and with whom they associated.

Some parents and professionals realized that when instruction was confined to the physical property of schools, too many important and learnable skills simply could not be acquired; the performance criteria used to define learning were inappropriate, counterproductive or even harmful if manifested in integrated nonschool settings; generalization difficulties disallowed the students from performing them appropriately in context in nonschool environments; and, even the best teachers and therapists ran out of good ideas quickly. Thus, it was arranged that a few would receive authentic vocational assessment and instruction during school days and times (Brown, Nisbet, et al., 1983).

Authentic vocational assessment refers to arranging for an individual to function in real integrated work and related environments and activities and then determining meaningful discrepancies between the repertoire of that individual and the minimally acceptable requirements of acceptable functioning therein. Authentic vocational instruction refers to teaching an individual that which is actually needed to meet the minimally acceptable performance standards of that real work environment. When first proposed, all but a few considered authentic assessment and instruction as radical, extreme, dangerous, impractical, too costly, developmentally absurd and creating false hopes. Those who argued for the status quo emphatically offered the following. "They will not be safe downtown. They should be with their own kind. You know how terribly she acts in the restroom at school; imagine if we take her to a public restroom. She always runs away. If we take her into the community, she will probably run under a bus. He is always touching others. That will not be tolerated." However, once the students demonstrated they could learn to function safely and efficiently in a variety of integrated settings, parents and teachers quickly realized that much more time should be devoted to generating the wonderful array of important skills that could only or best be acquired from direct instruction therein.

After a few years, stories of success spread and more teachers realized they could also teach meaningful skills, increase the number of environments in which their students functioned and develop constructive social relationships with individuals without disabilities. As more teachers participated, more students were given opportunities and more parents wanted integrated nonschool instruction for their children. ${ }^{1}$

In the early 1980's students who received direct instruction in integrated work and related settings as part of their curricula approached school exit. Their parents, teachers and others were extremely frustrated, disappointed and angry about the limited post school options. Several worked collaboratively with Dane County, WI professional and elected officials to bypass the sheltered workshops, activity centers and waiting lists by assigning public funds to agencies that would support the students in the integrated work settings in which they were functioning at school exit.

This is a report of 50 workers who exited school from 1981 to 2003 and who functioned in integrated work settings from 1981 to 2020. Specifically, information is presented pertaining to the work environments utilized, the work tasks performed, the major reasons for changes in environments and tasks, the hours worked per week, the wages earned, the travel modes used to get to and from work, the extra supports provided by Job Coaches, involvement in Community and Recreation activities during work days and times and where each resided. Social relationships with coworkers without disabilities, the mortality of the 50 workers and their parents and guardianship are also addressed.

Certainly, selected individuals with significant intellectual disabilities have functioned in integrated work settings for centuries and many do today. (Blackorby \& Wagner, 1996; Certo et al., 1997, 2002; Horvath, Rose \& Stapleton, 2003; Luecking \& Certo, 2003; Murphy \& Rogan, 1995; PCESE, 2002; PCID, 2004; Wehman \& Kregel, 1998). Nevertheless, far too many exit schools and are confined to segregated

\footnotetext{
${ }^{1}$ Funds from the USDOE, Bureau for the Education of the Handicapped, Special Projects provided valuable support for these activities. An expanded version of this report is available on the CWS website www.cwsmadison.com.
} 
enclaves, workshops and activity centers or stay in residences all day with family members and persons who are paid to be with them. Hopefully, this report can assist those interested in changing these most unfortunate school outcomes.

\subsection{Community Work Services Inc. (CWS)}

CWS was established in 1984 by Betsy Shiraga and Kim Kessler. From its inception it received public funds to serve adults with significant intellectual and related disabilities in integrated work environments. In 2011 Betsy Shiraga and Kim Kessler retired. Sarah Cutler, who had been a Job Coach for five years, became the Executive Director and Sharon Schmid, who had been a Job Coach for 20 years, became the Program Director. In January, 2020 CWS served 79 workers, employed an 80\% time Executive Director, a full time time Program Director, a 50\% time Office Manager and the equivalent of 16.5 full time Job Coaches. The total amount of public funds received in 2019 was $\$ 1,245,359$. This converts to an average cost of $\$ 15,764$ per year per worker.

\section{Data sources and methodology}

The Community Work Services Cumulative Worker Record (Cutler \& Schmid, 2020) was developed to capture most of the information contained in this report. The Executive Director and the Program Director then informed Job Coaches how they were to use the record to compile information about the workers for whom they were directly responsible. When a Job Coach gathered the necessary information, he /she met with at least one of the authors and completed each record as thoroughly as possible. The primary data sources were the cumulative file of a worker, recollections of the present and former Directors, information gathered from parents and guardians, CWS Annual Reports, written and verbal reports of Job Coaches and information gathered from the workers, employers, coworkers without disabilities and residential personnel. In some instances, all the information necessary was recorded. In others a small amount of information was not available or unknown.

After the cumulative records were completed, Tables $1 \& 2$ were constructed and much of the information gathered was presented therein. An earlier report of these workers addressed their functioning from 1981 through 2005 (Brown, Shiraga \& Kessler,
2006). As this 2020 report is an extension of that one, in order to maintain a continuous record, it was necessary that much of the information contained in it be repeated here. In addition, information related to guardianship and mortality were added.

Confidentiality procedures were approved by the workers through their parents or guardians, as well as the Executive Director, the Program Director and the CWS Board of Directors. Numbers and pseudonyms rather than actual names were used.

\subsection{The workers}

In 2005 CWS served 70 workers. Forty had received instruction in integrated work and related environments while they attended MMSD schools and were served by CWS at school exit. Seven exited the MMSD before CWS was established. They had received instruction in integrated work and related environments while they attended school and were served by local agencies at school exit. They moved to CWS because their parents and/or their Dane County Case Managers requested they do so. Three did not attend MMSD schools and did not receive instruction in integrated work and related environments while they attended schools in Dane County. They were referred to CWS because of the preferences of their parents or Dane County Case Managers. Nine were long term residents of state operated institutions for the Developmentally Disabled. When they were relocated to residential settings in Madison, Case Managers referred them to CWS, who then arranged for them to function in integrated work settings. Eleven had been functioning in activity centers or a local sheltered workshop. Their parents or Case Managers requested they be served by CWS.

When the 2006 study was designed, the authors decided to focus on all 47 individuals who had received instruction in integrated work and related environments during their school careers. The three who attended three high schools in Dane County, but did not receive instruction in integrated work and related environments, were selected because they had recently exited or were in the process of exiting school, they functioned in the same range as the other 47 selected and they allowed the preferred sample size of 50 .

Demographic and diagnostic information is presented in Table 1 - Columns A and B. The 50 workers include 21 females. Forty five were Caucasians, three were African Americans, one was Latino and one was Asian American. Forty were reported as having 


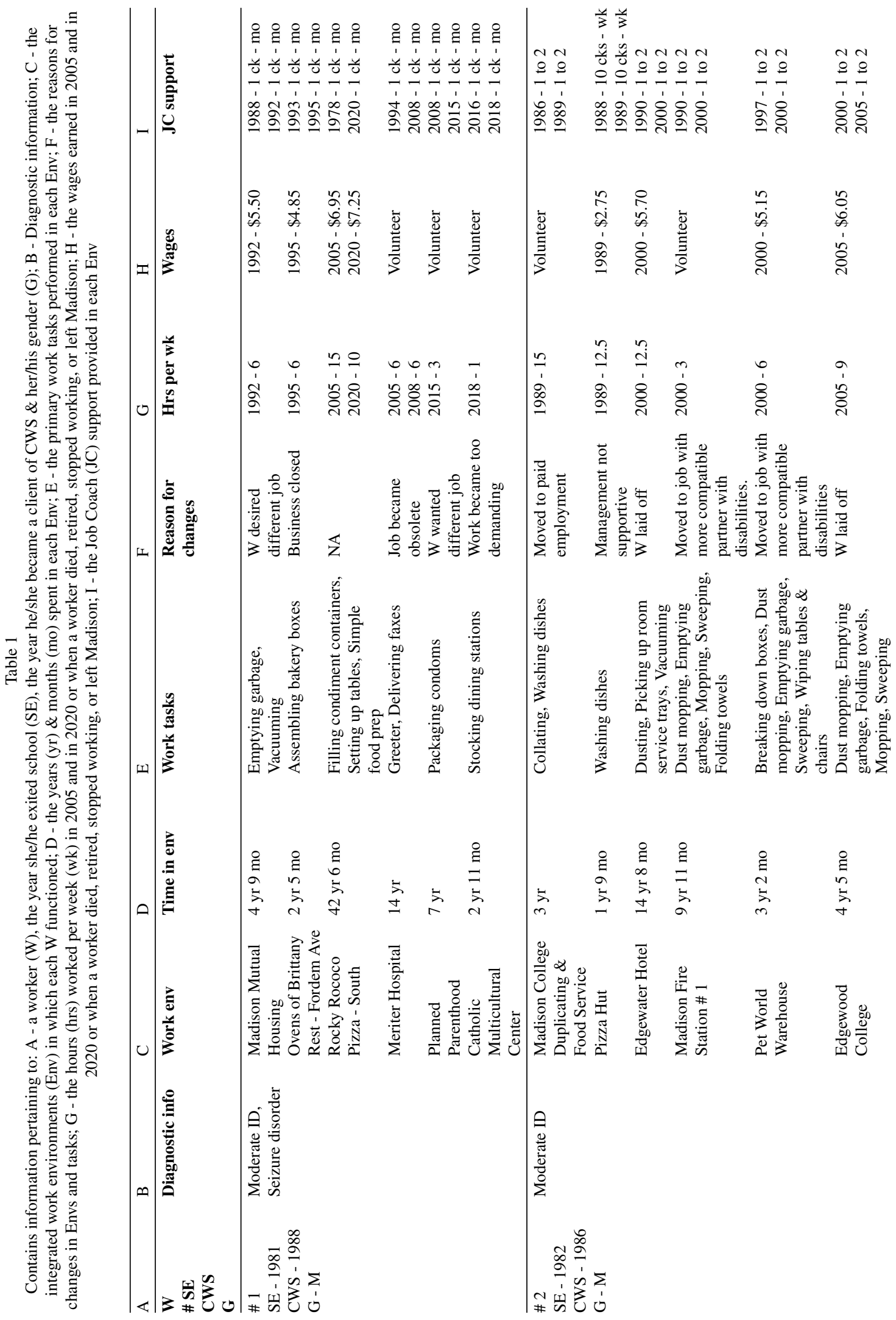




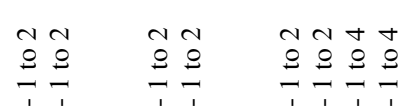

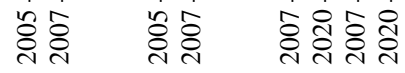

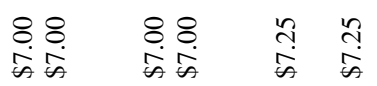

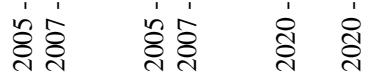

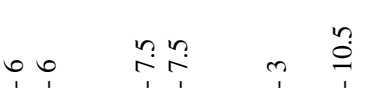

官客

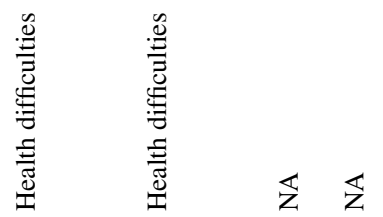

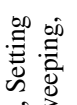

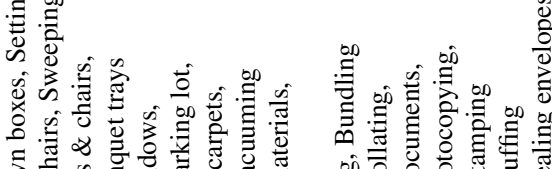

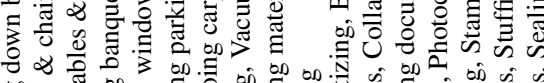

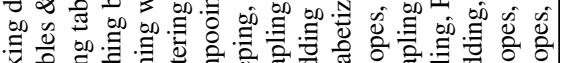

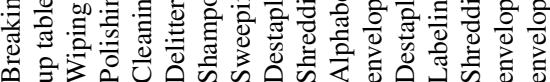

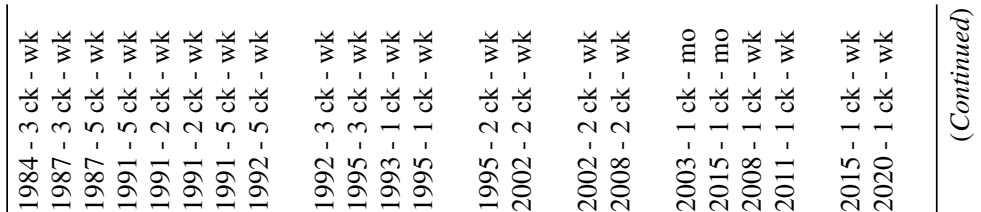

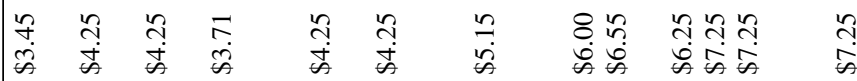

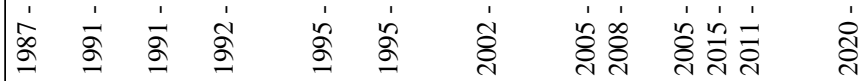

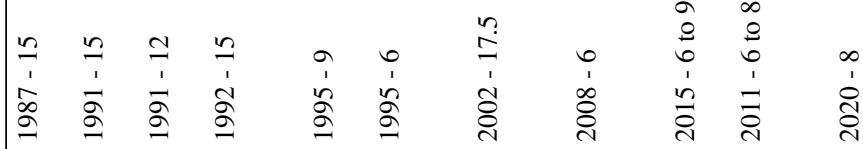

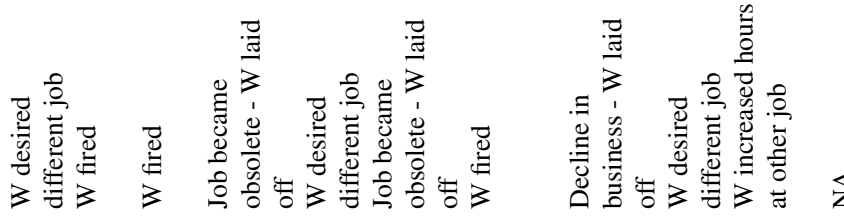

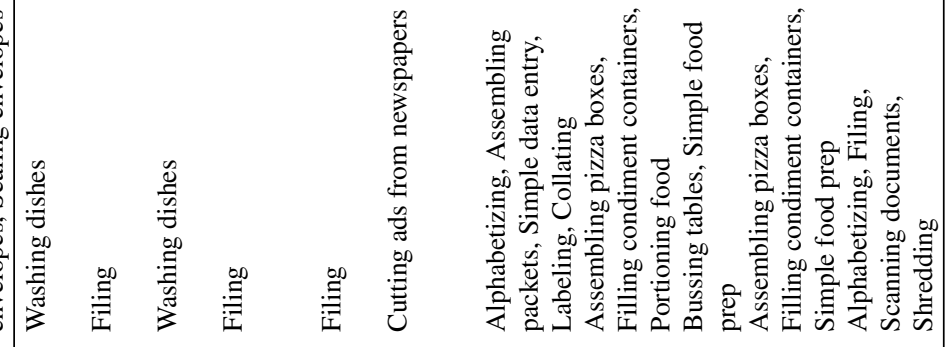

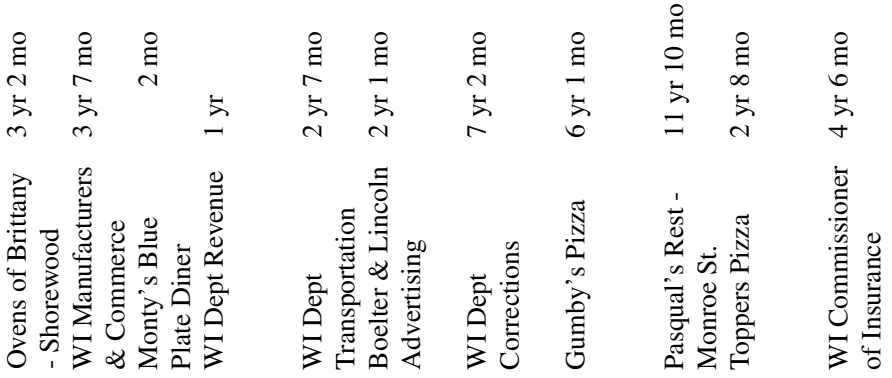

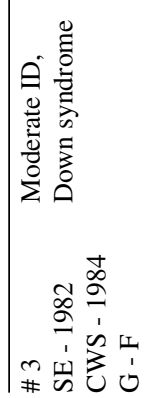




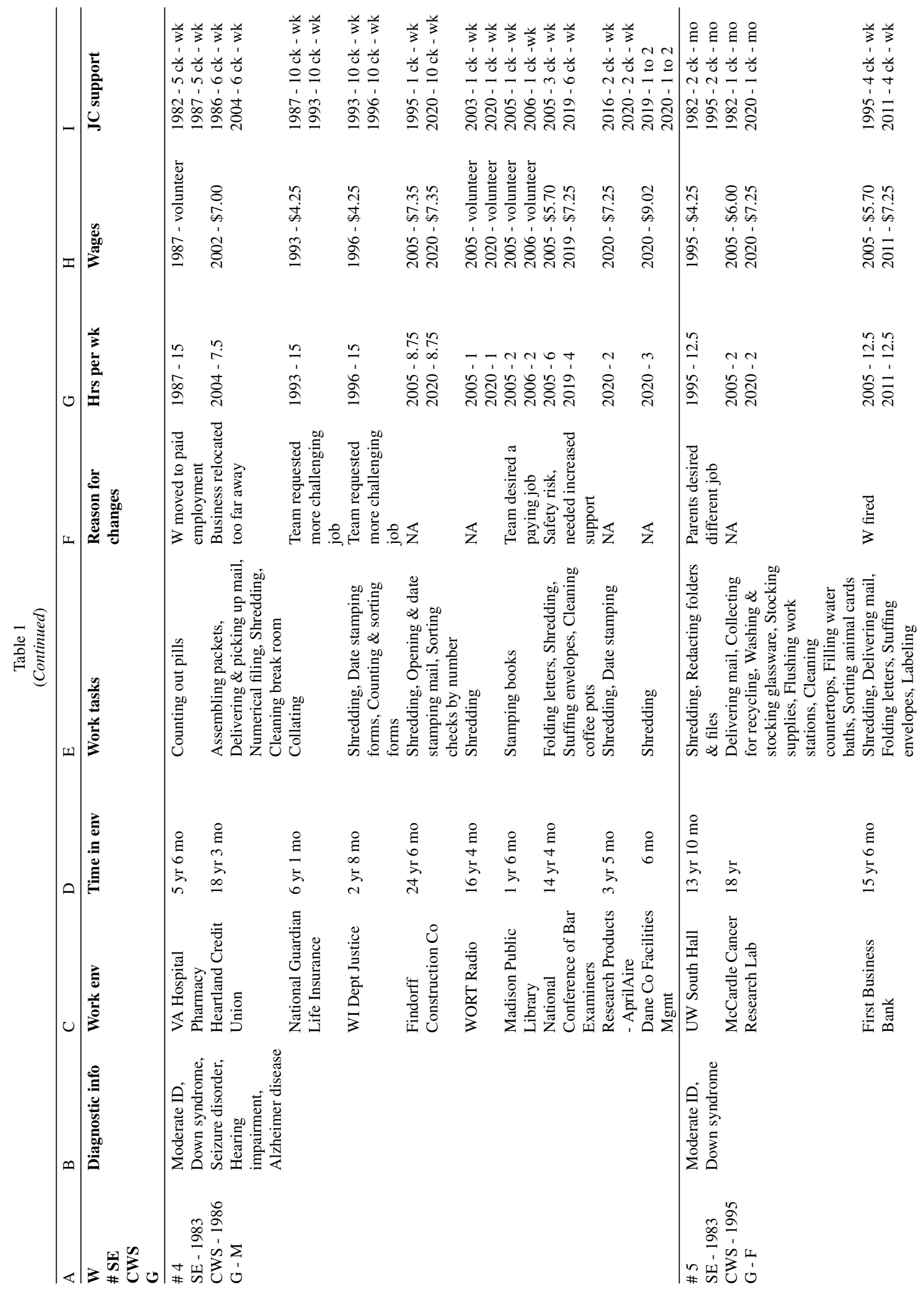




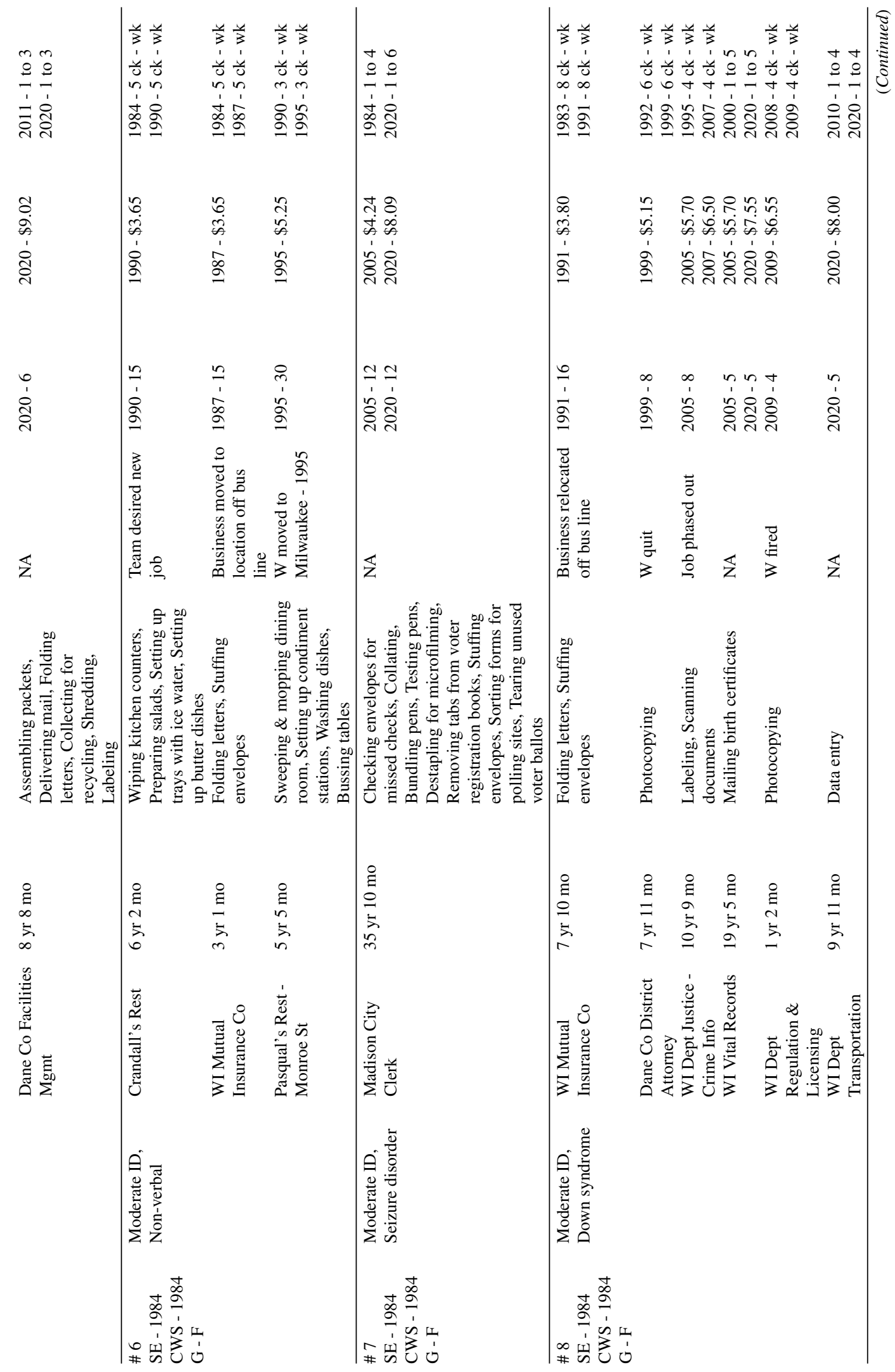




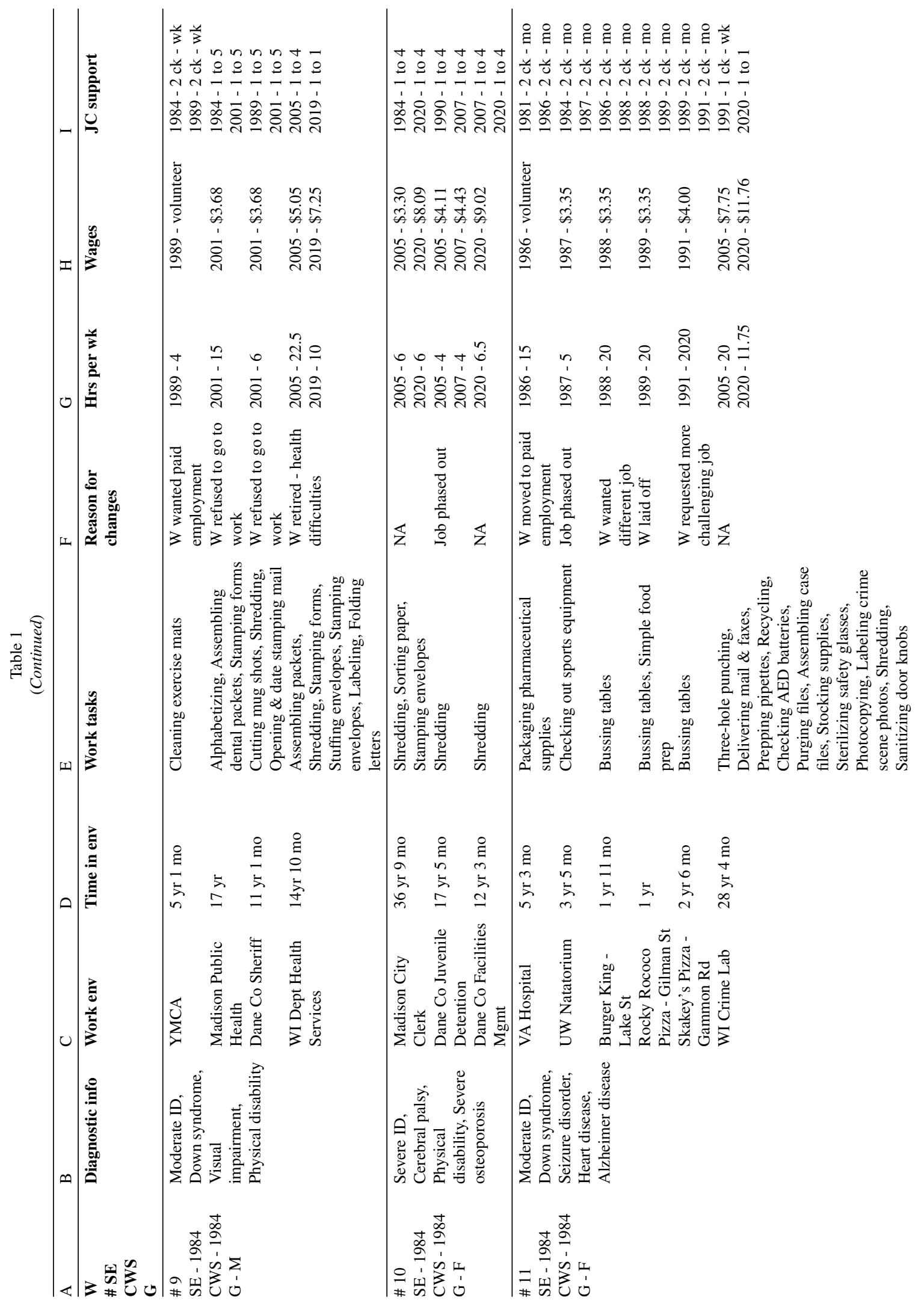




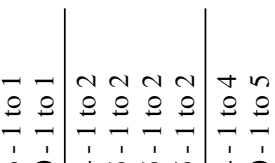

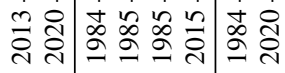

$\stackrel{1}{2} \stackrel{1}{9} \stackrel{1}{9}$

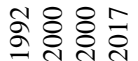

을

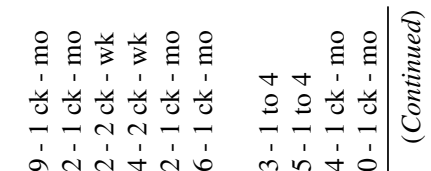

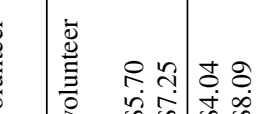

-

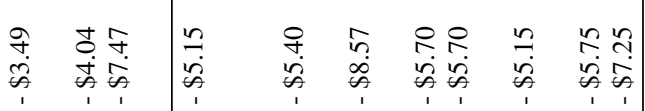

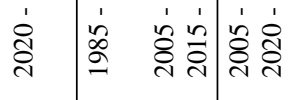

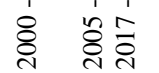

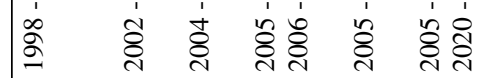

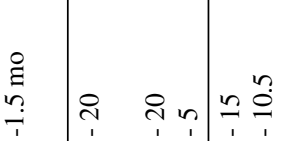

商

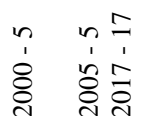

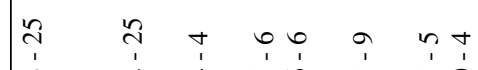

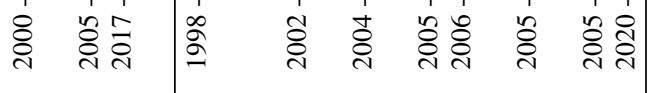

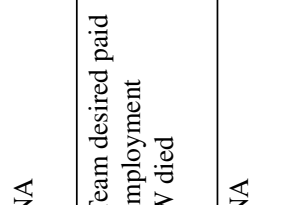

ㅇํㄹ

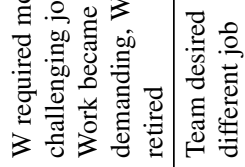

उ

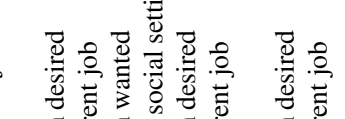

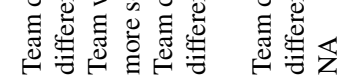

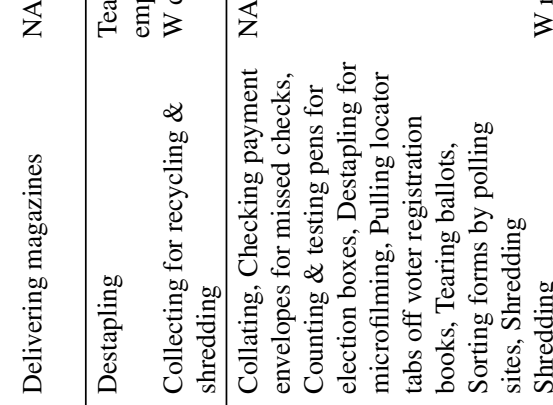

(1)

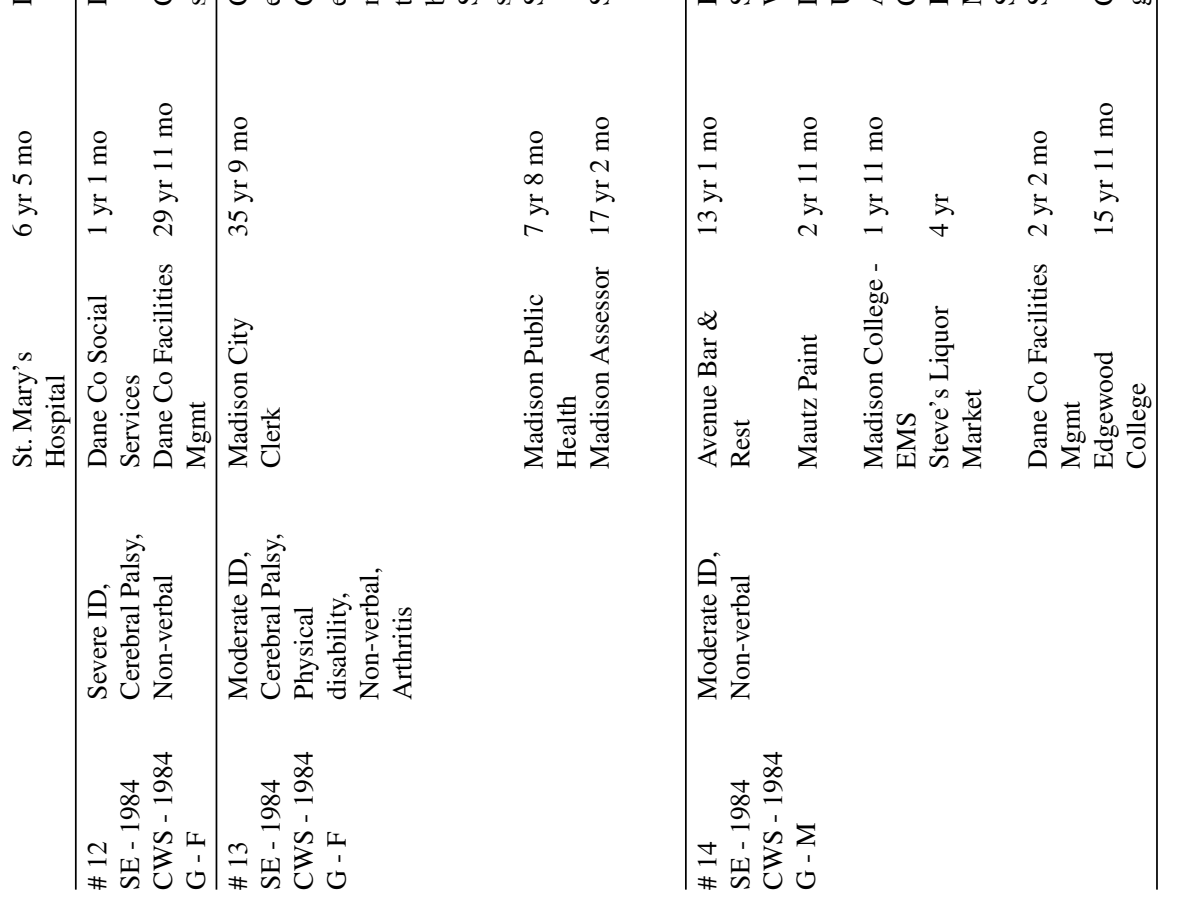




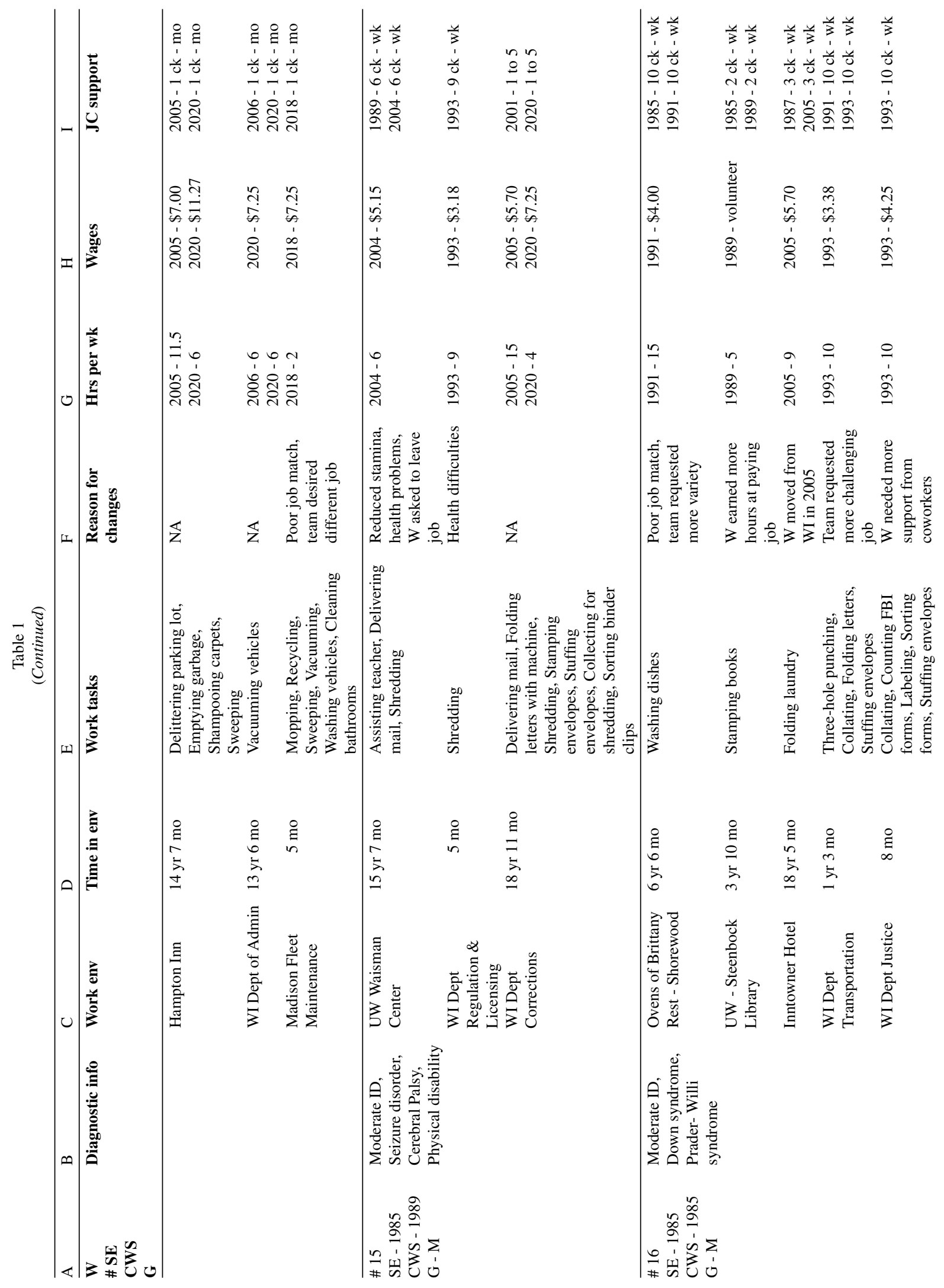


要

$\frac{4}{3} \frac{4}{3}$

in $\ln 00$

过

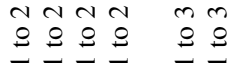

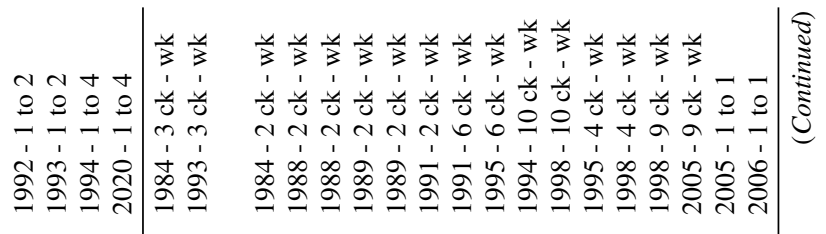

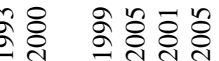

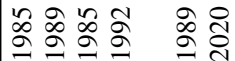

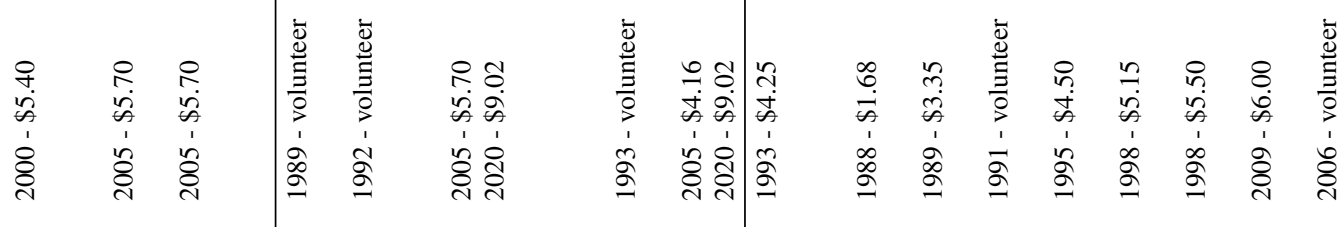

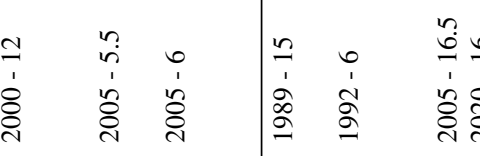

0

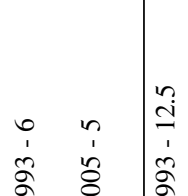

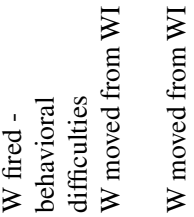

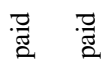

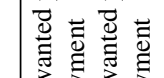

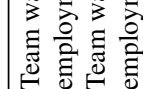

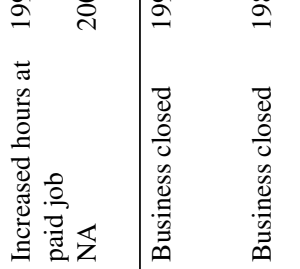

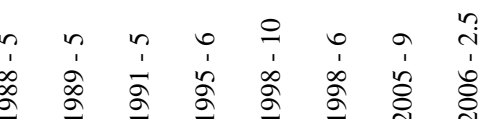

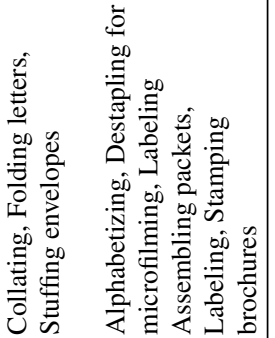

蓆

宽

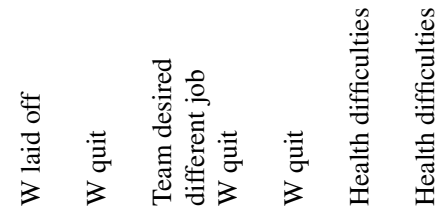

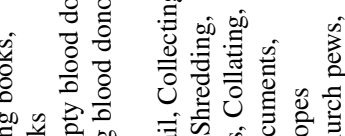

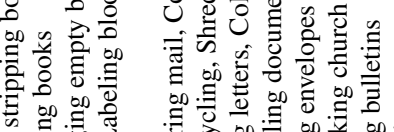

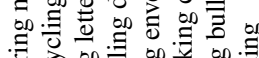

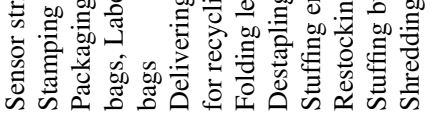

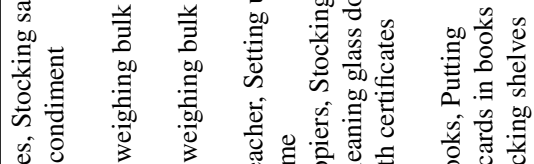

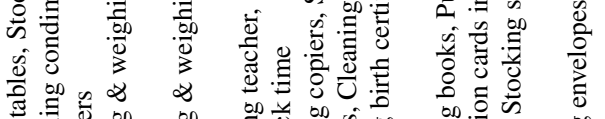

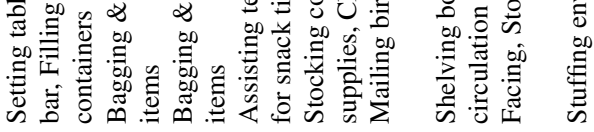

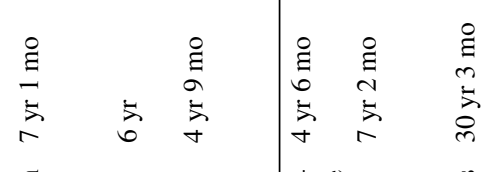

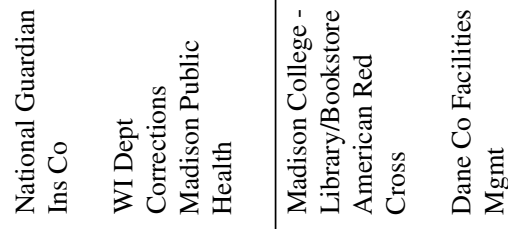

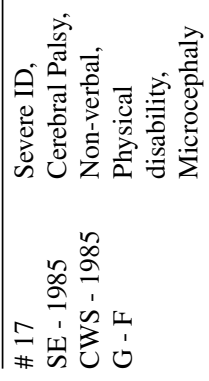

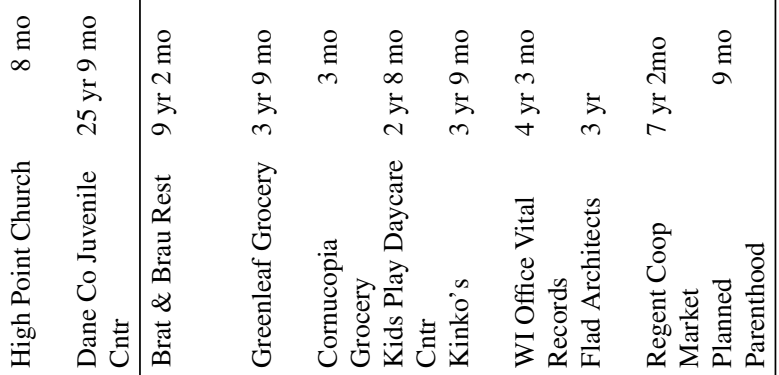

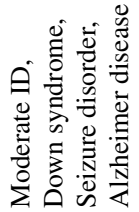

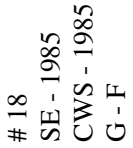




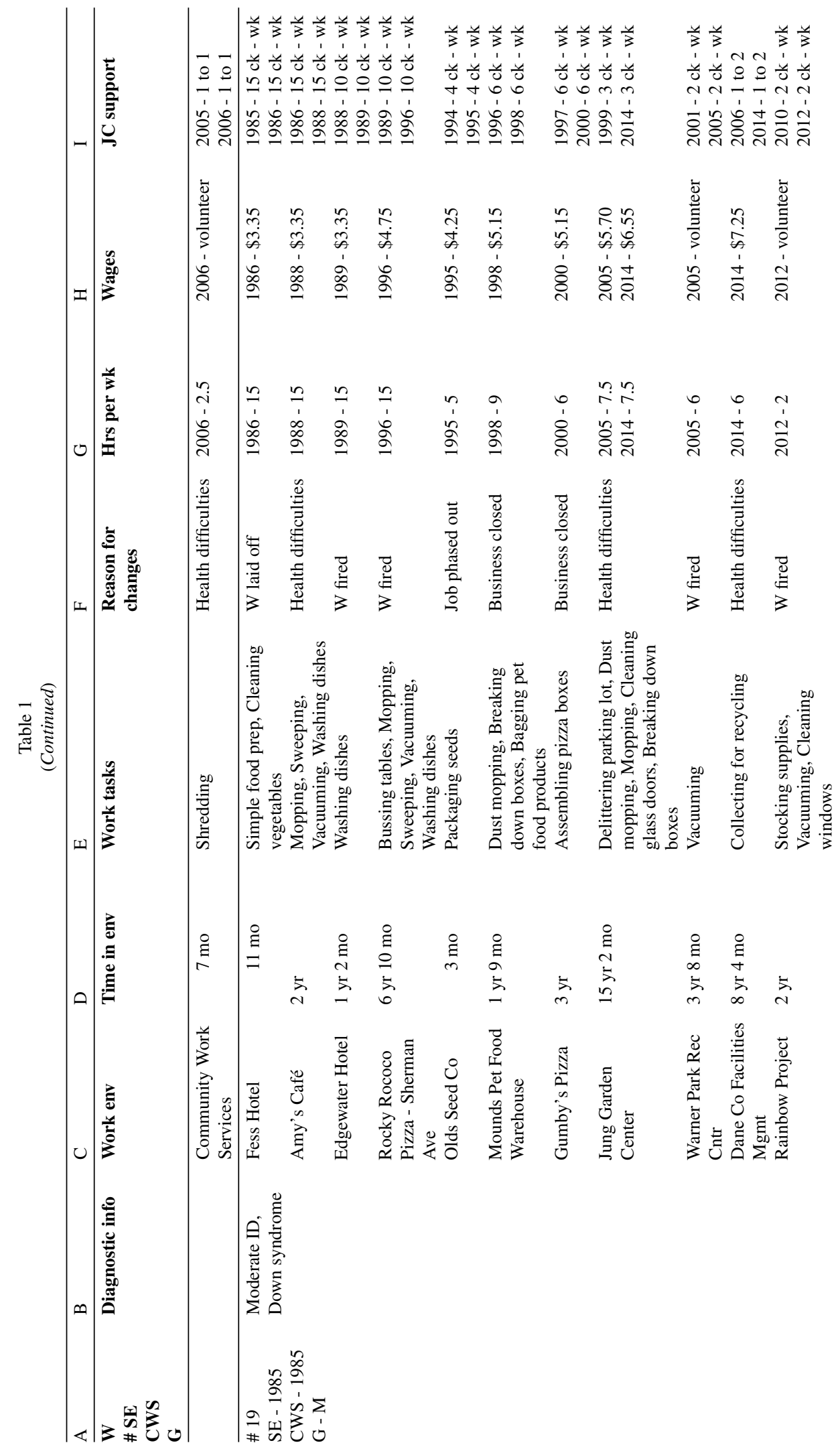




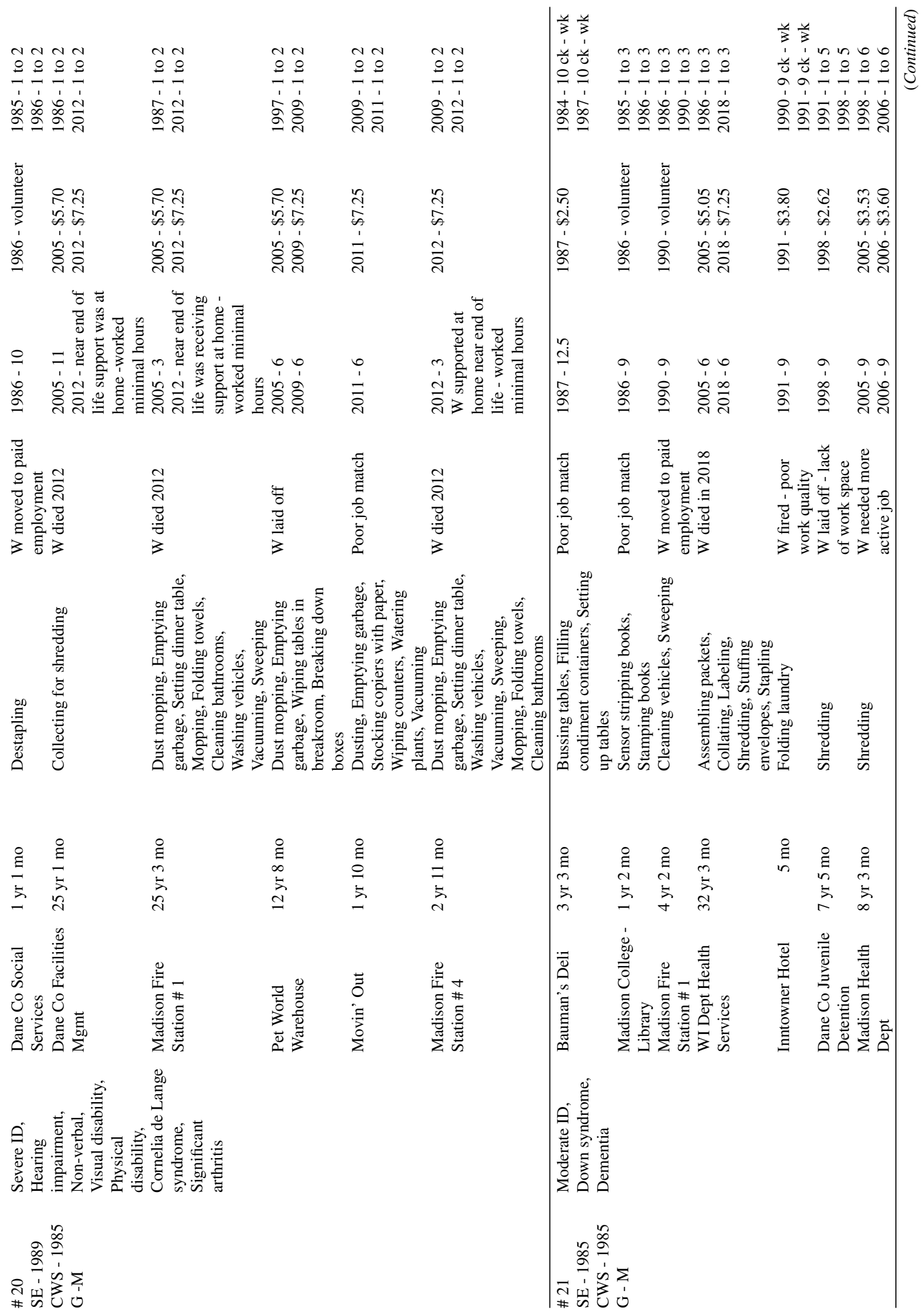




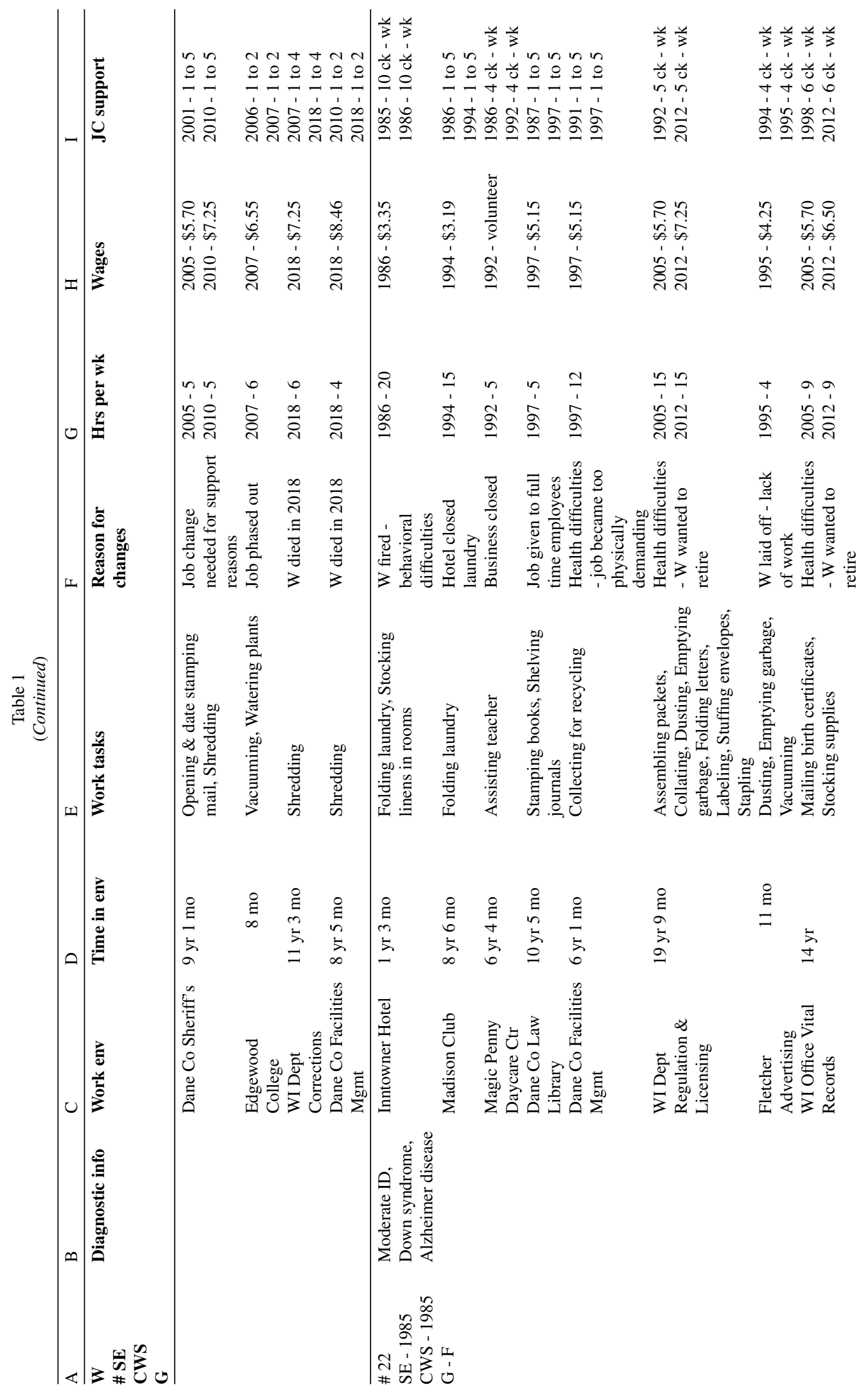




\begin{tabular}{|c|c|c|c|c|c|c|c|c|c|c|}
\hline 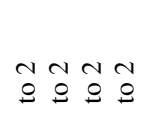 & $\stackrel{m}{\varrho} \stackrel{m}{g}$ & 롱 & $\stackrel{5}{g} \stackrel{5}{9}$ & $\begin{array}{ll}m & m \\
9 & 9\end{array}$ & 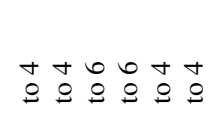 & 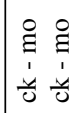 & $\stackrel{+}{g} \stackrel{+}{g}$ & $\begin{array}{l}\frac{3}{3} \\
\frac{3}{3} \\
\frac{1}{3} \\
\frac{1}{3}\end{array}$ & $\stackrel{N}{g} \mathscr{g}$ & $\begin{array}{ll}n & n \\
2 & 8\end{array}$ \\
\hline 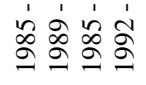 & 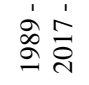 & ๙ิ่ & 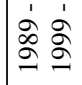 & 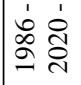 & 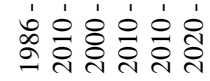 & 定 & 宫 & 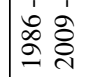 & 家 & 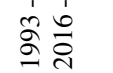 \\
\hline 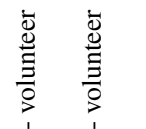 & 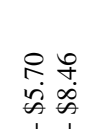 & 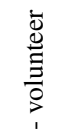 & $\frac{n}{2}$ & 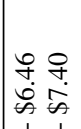 & 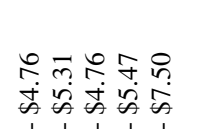 & $\begin{array}{ll}8 & m \\
0 & \infty \\
0 & \infty \\
1 & 0\end{array}$ & $\begin{array}{l}8: \\
0 \\
0 \\
0 \\
0\end{array}$ & 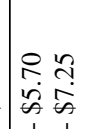 & $\begin{array}{l}n \\
2 \\
\vdots \\
0\end{array}$ & $\begin{array}{l}n= \\
\alpha \\
w \\
b \\
b\end{array}$ \\
\hline$\stackrel{\circ}{\circ} \stackrel{\alpha}{\sigma}$ & 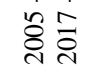 & $\hat{\sigma}$ & פे & ) & 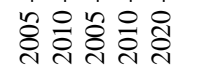 & 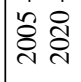 & 完 & 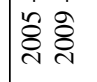 & \& & 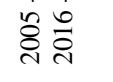 \\
\hline 0 & 주주 & 0 & i & $\because 0$ & agmmo & 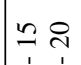 & th & 00 & $n$ & \\
\hline$\stackrel{\infty}{\circ}$ ఏ & ठั่ & $\stackrel{\check{\sigma}}{2}$ & gे & | & ڤั่ & 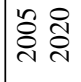 & \&ें & 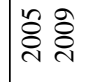 & $\stackrel{\circ}{\circ}$ & 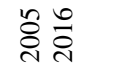 \\
\hline 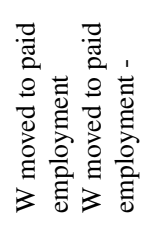 & 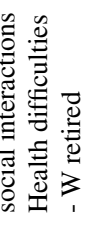 & 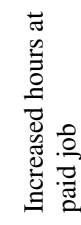 & 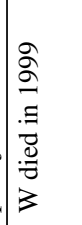 & $\mathbb{z}$ & 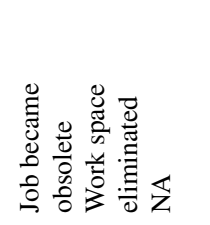 & $\mathbb{z}$ & 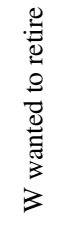 & 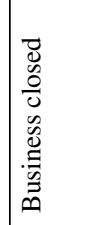 & 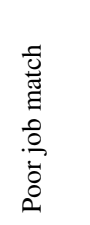 & 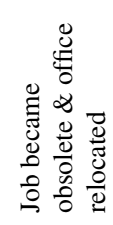 \\
\hline
\end{tabular}

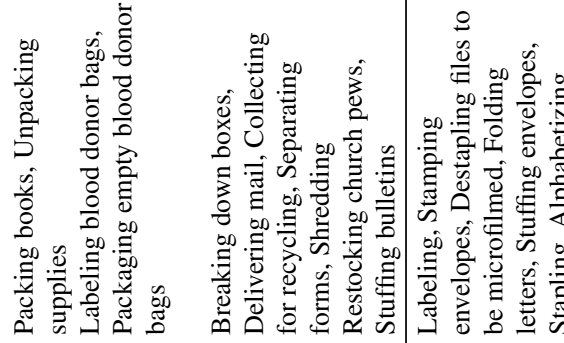




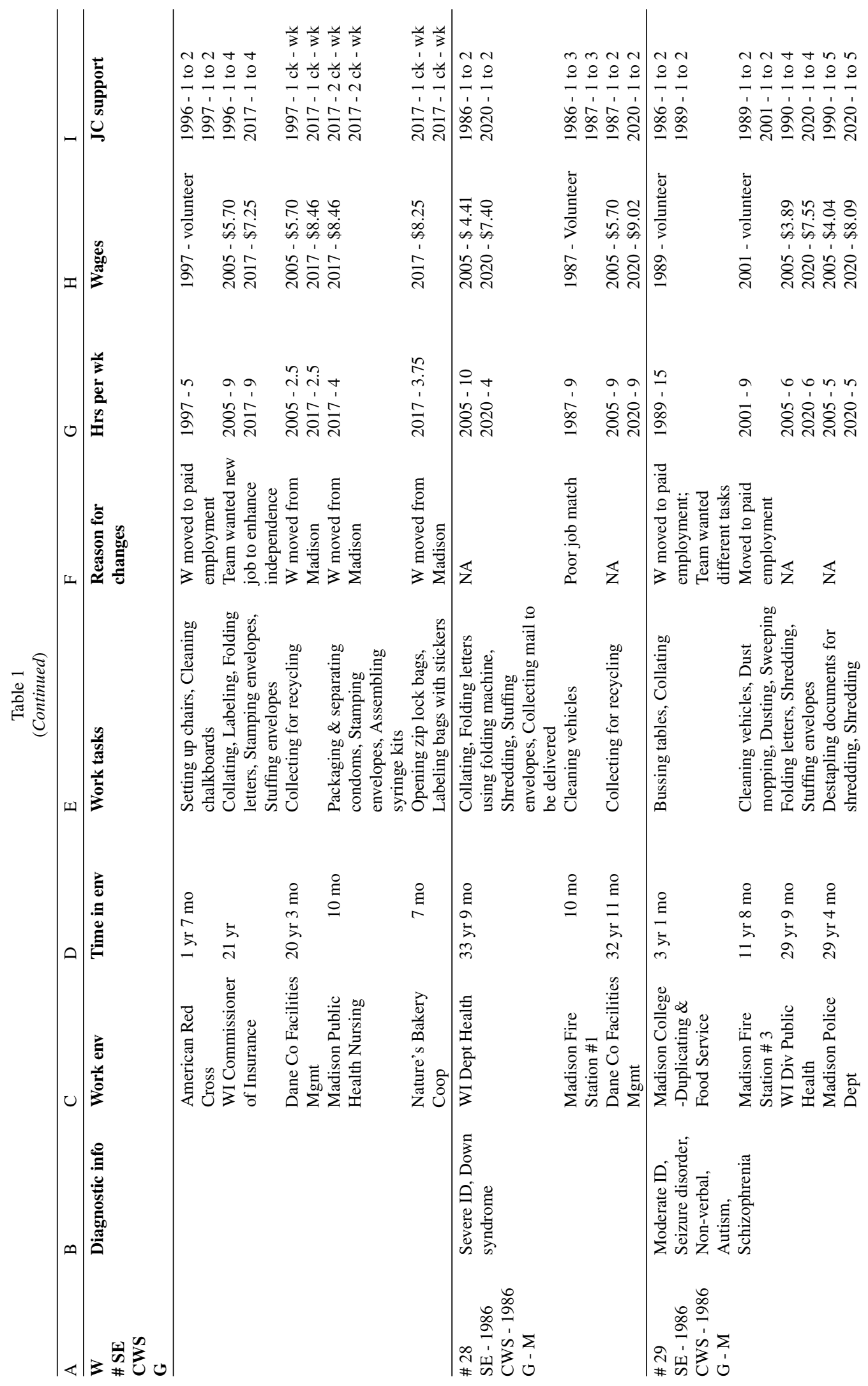




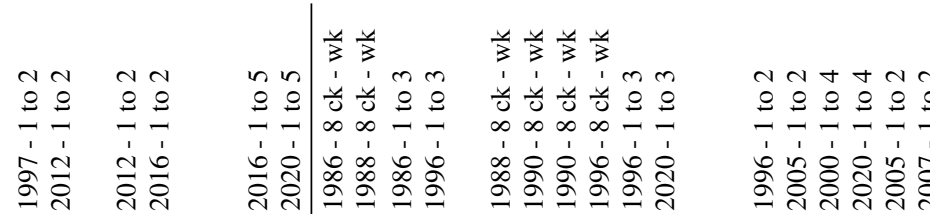

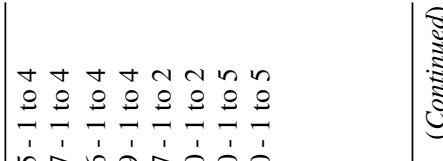

\%

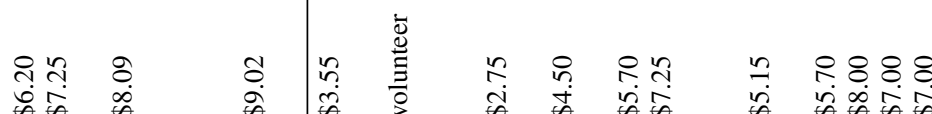

นิ

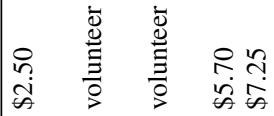

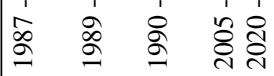

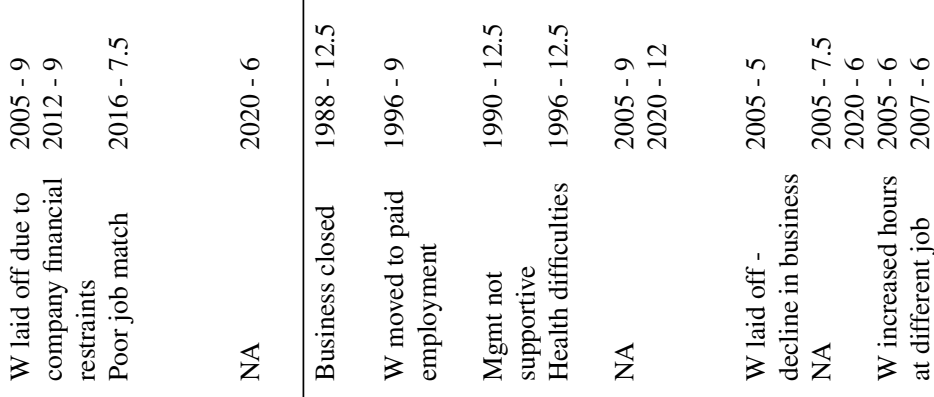

i in a $\stackrel{n}{?} ?$

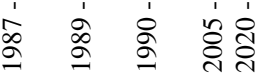

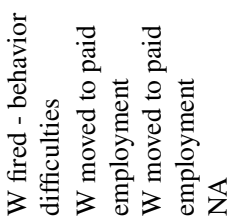

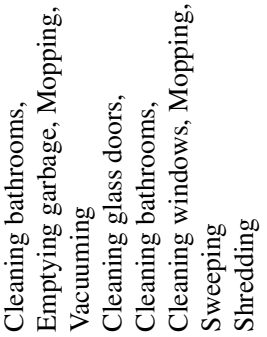
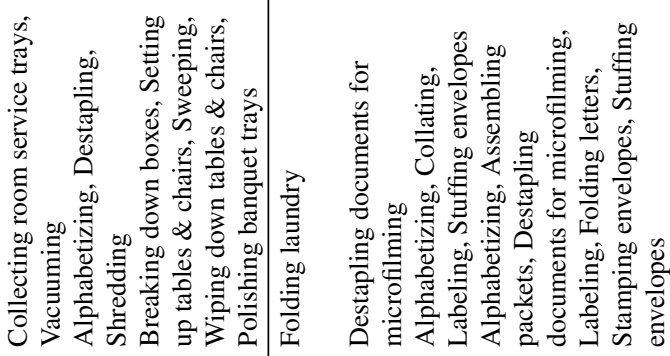

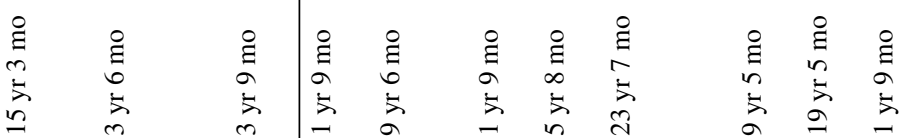

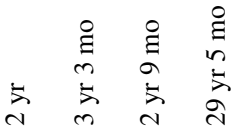

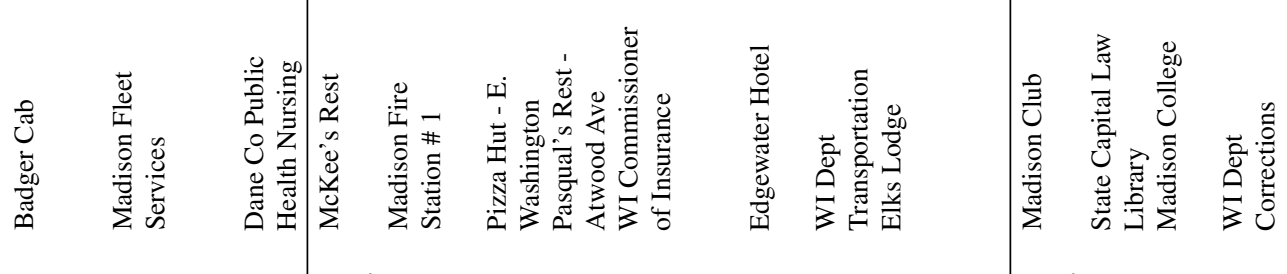

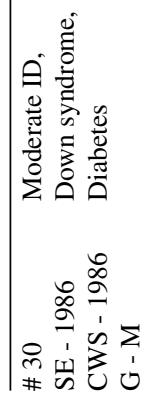

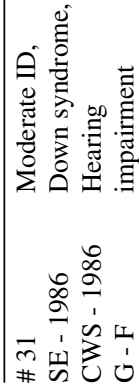




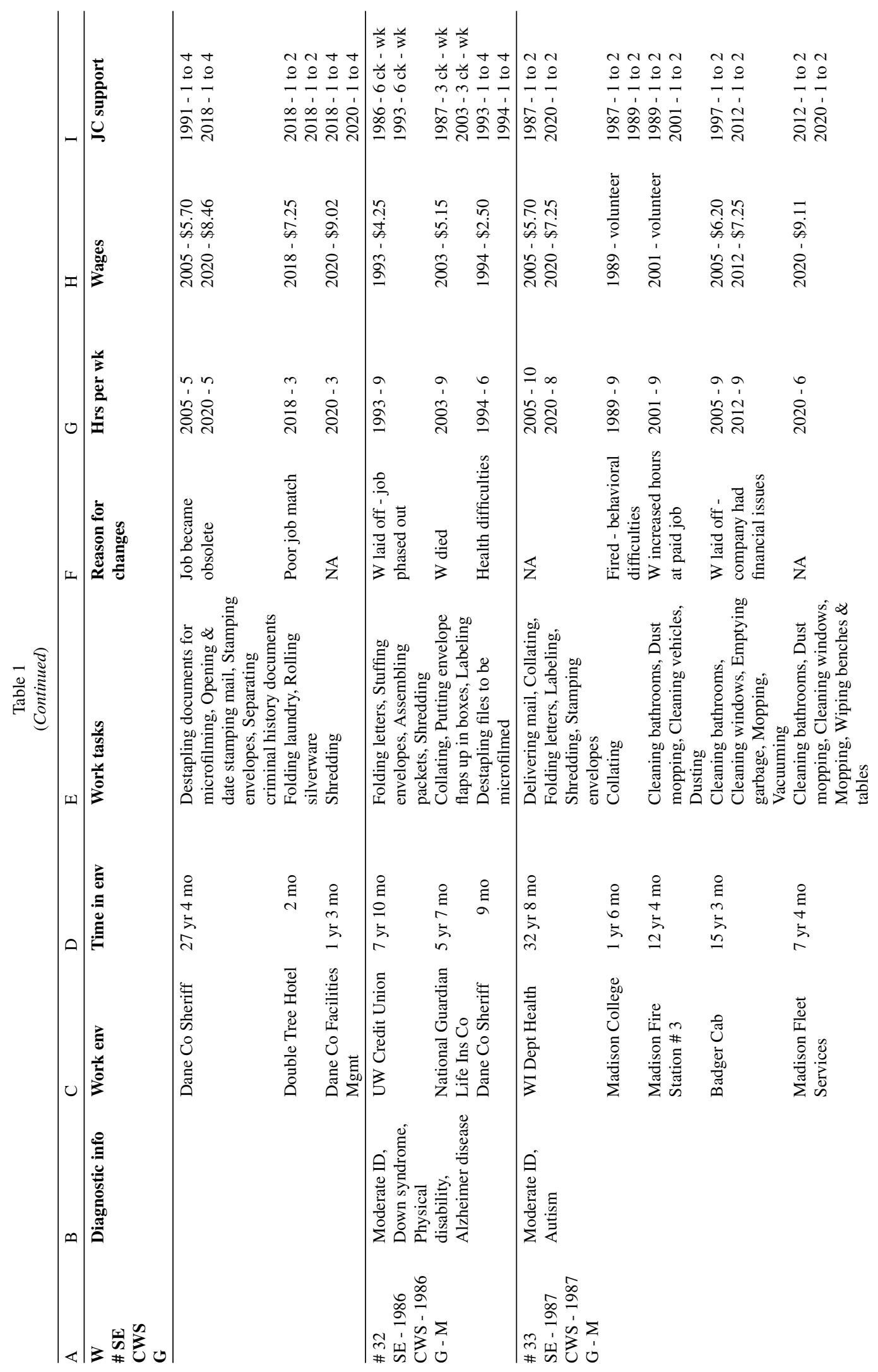


$\frac{4}{3} \frac{4}{3}$

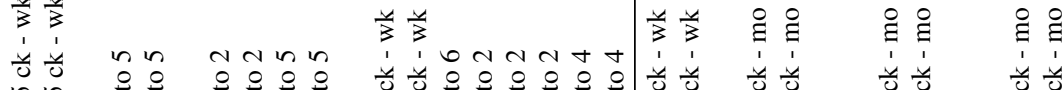

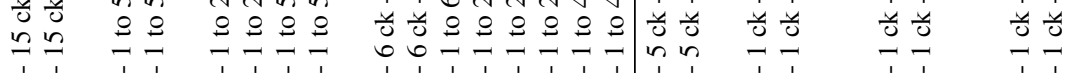

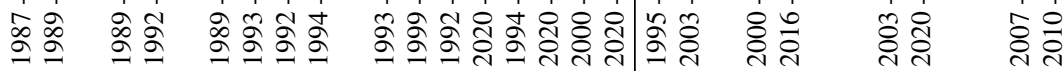

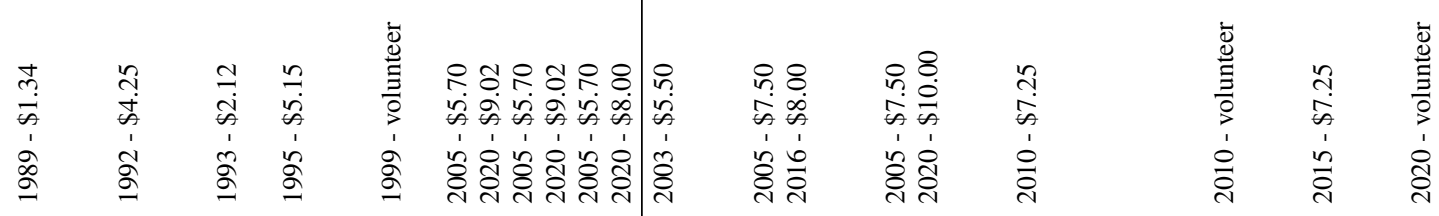

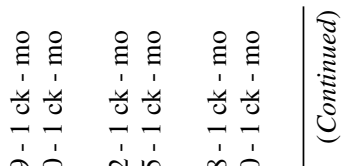

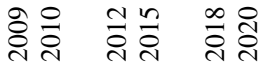

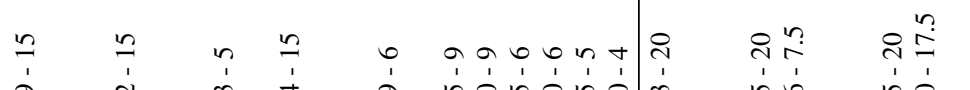

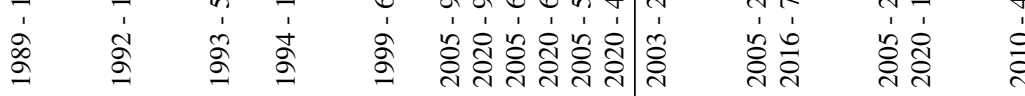

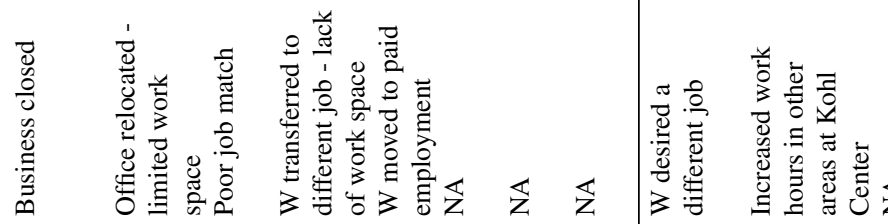

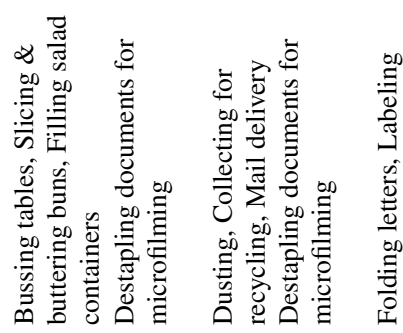

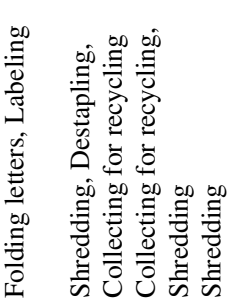

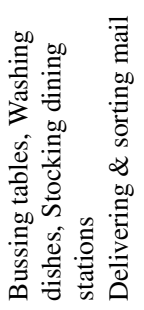

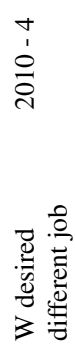

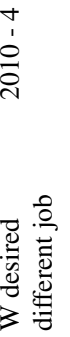

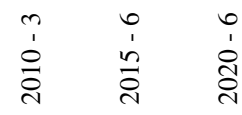

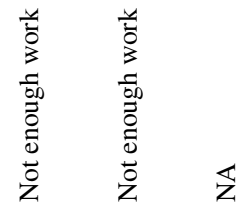




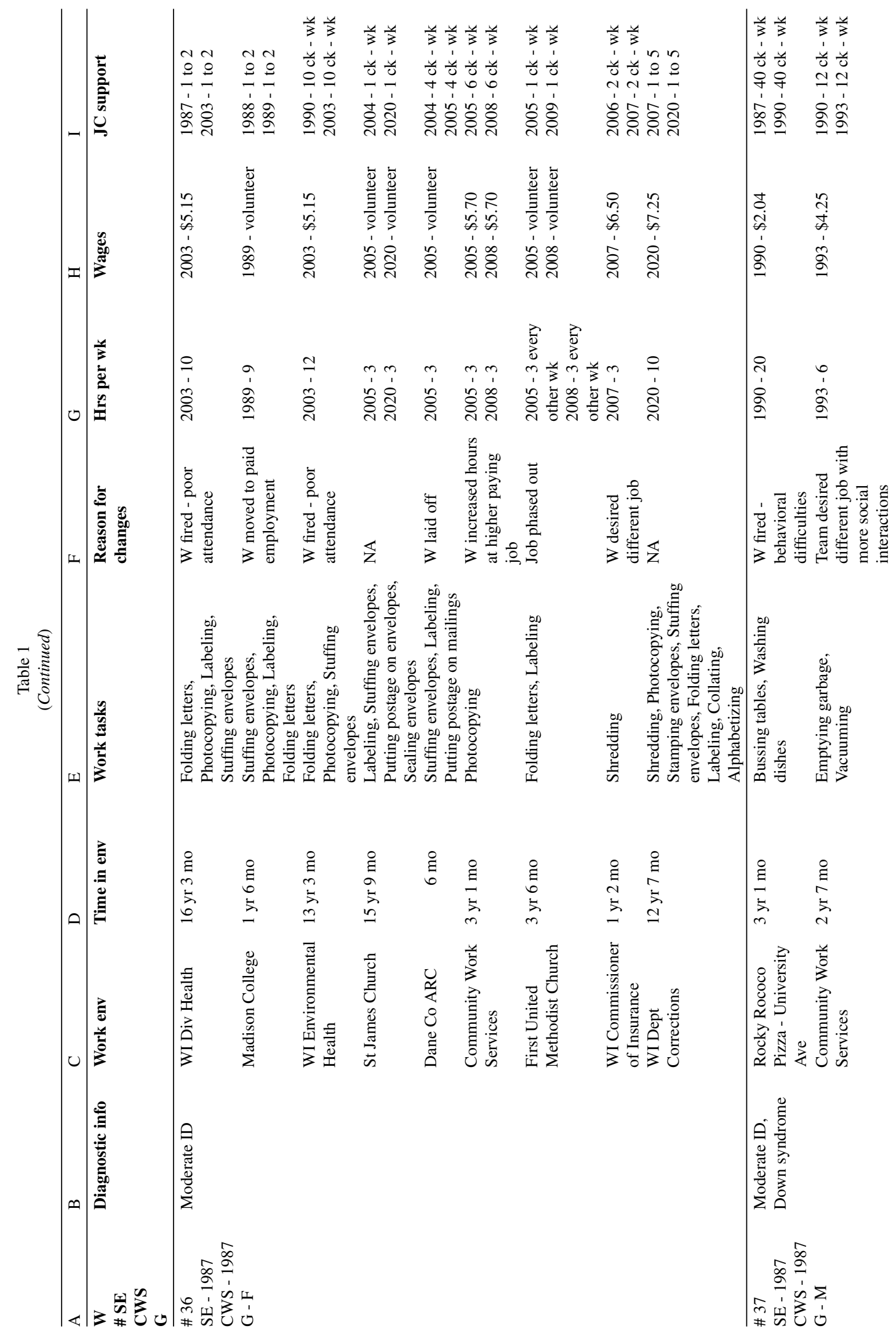


$\frac{4}{3} \frac{4}{3} \quad \frac{4}{3}$

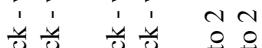

$\begin{array}{lllll}0 & 0 & 0 & 0 & 0 \\ 0 & 0 & 0 & 0 & 0\end{array}$

ํำ

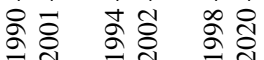

ஃ。

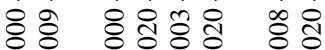

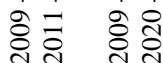

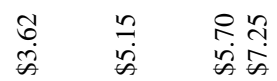

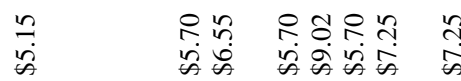

$\stackrel{n}{\infty} \stackrel{n}{n}$

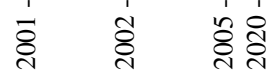

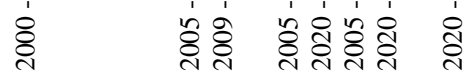

ङ

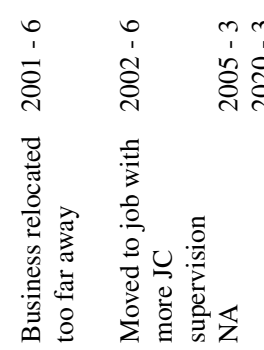

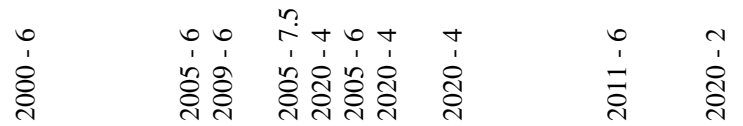

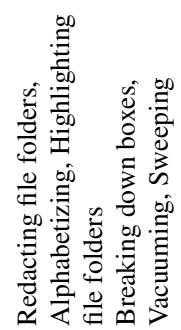

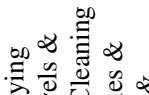

$\frac{3}{\frac{3}{3}}$

$\frac{7}{3}$

$\overleftrightarrow{z} \mathbb{z}$

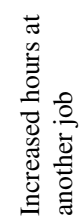

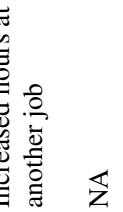

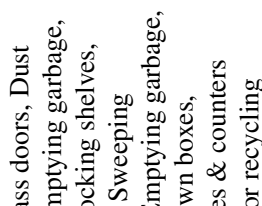

要

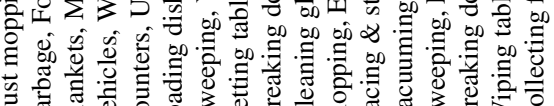

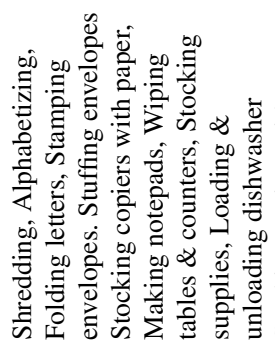

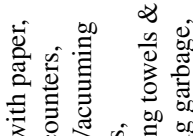

0 $\infty$

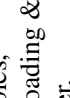

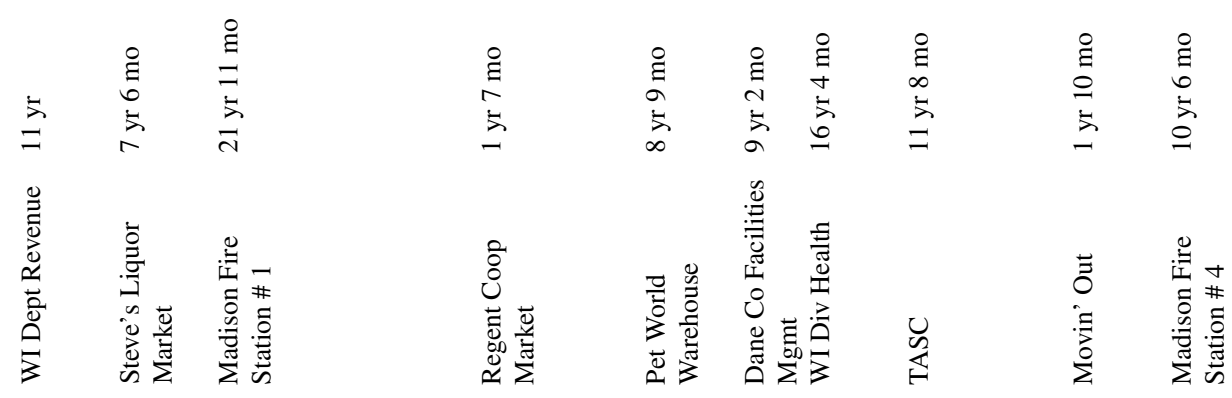




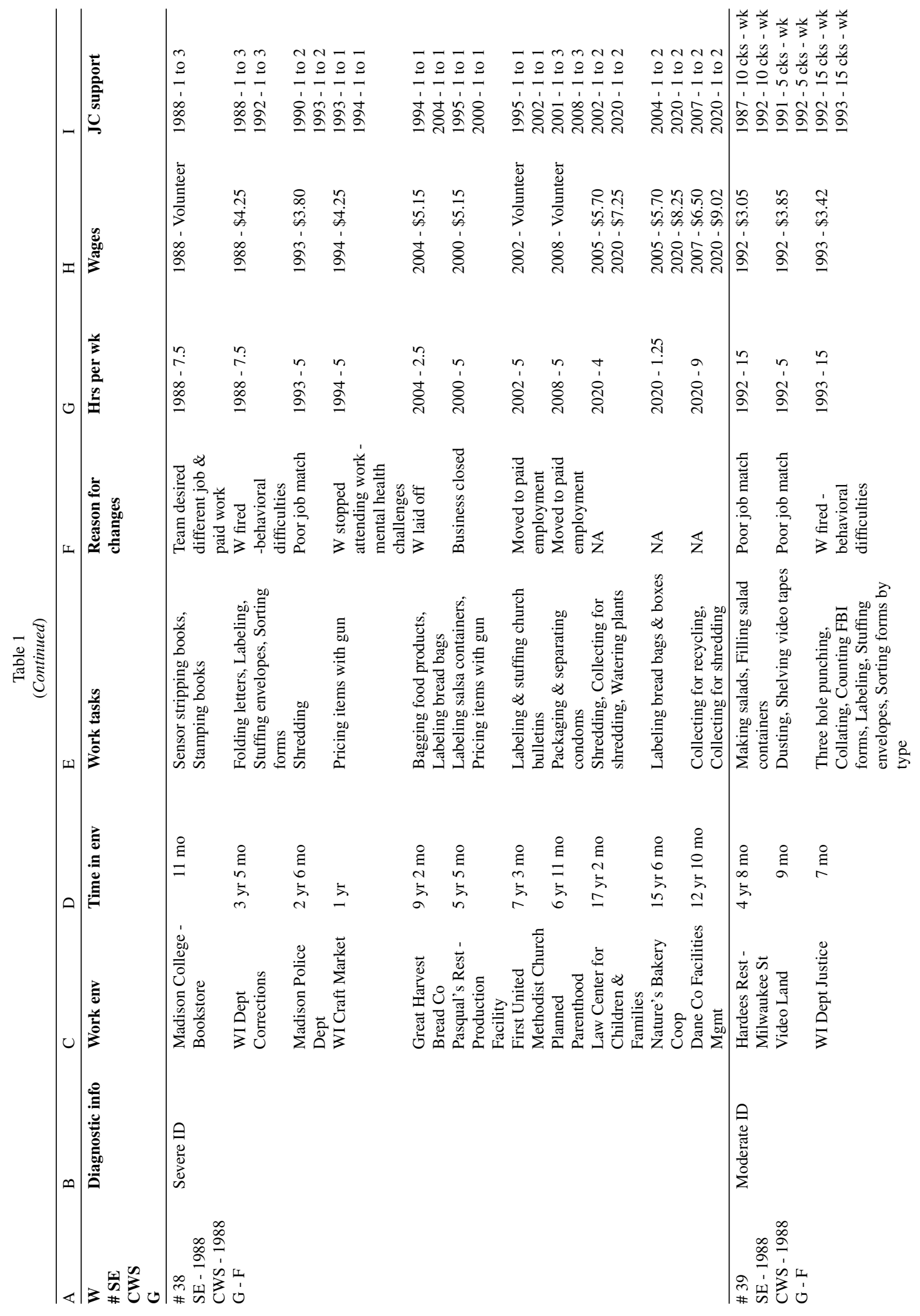




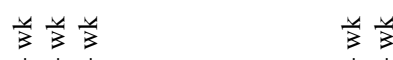

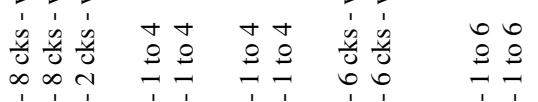

बूँ

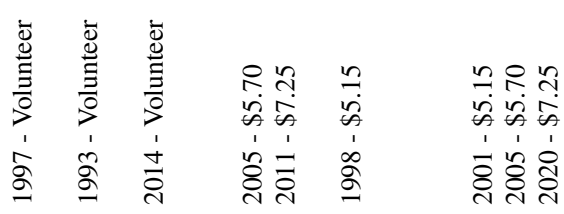

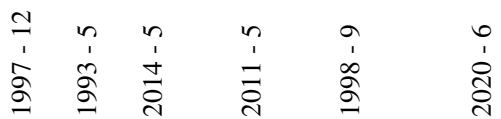

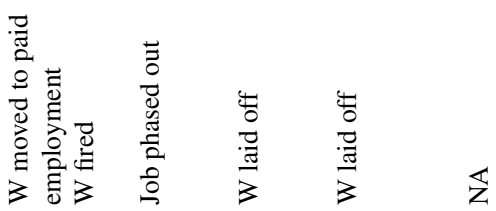

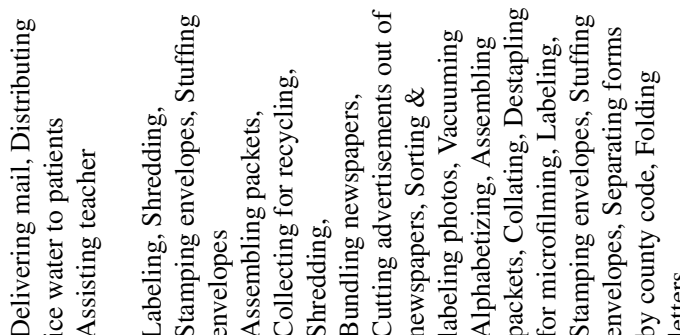

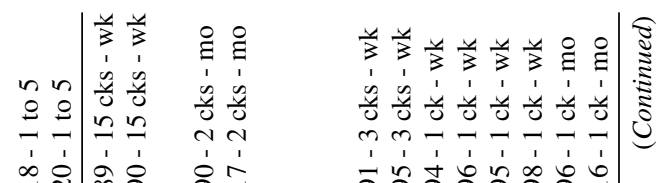

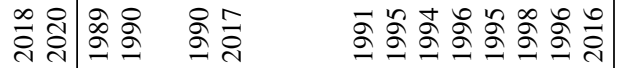

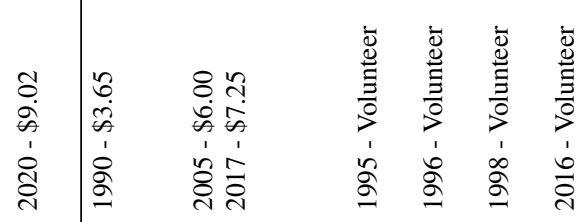

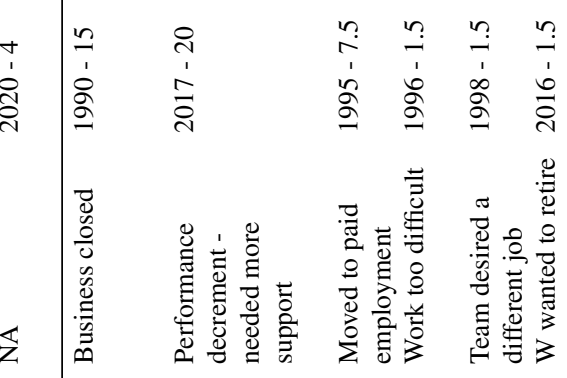

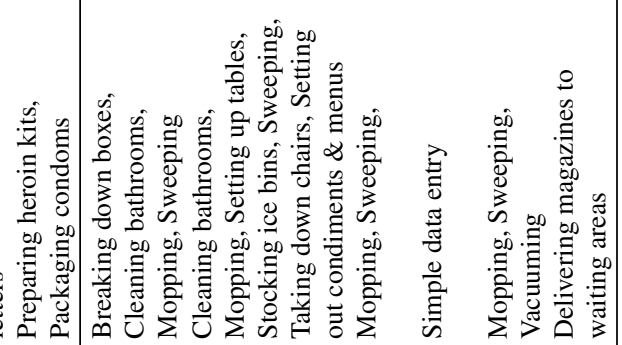

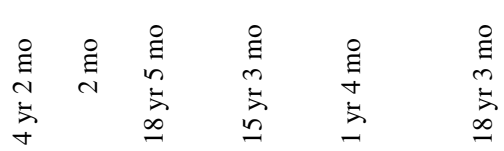

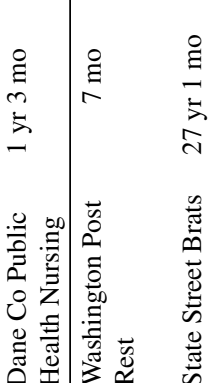

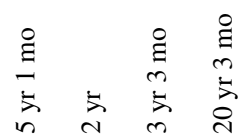

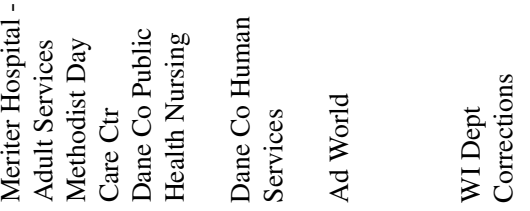

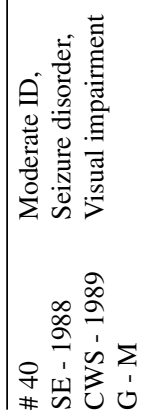




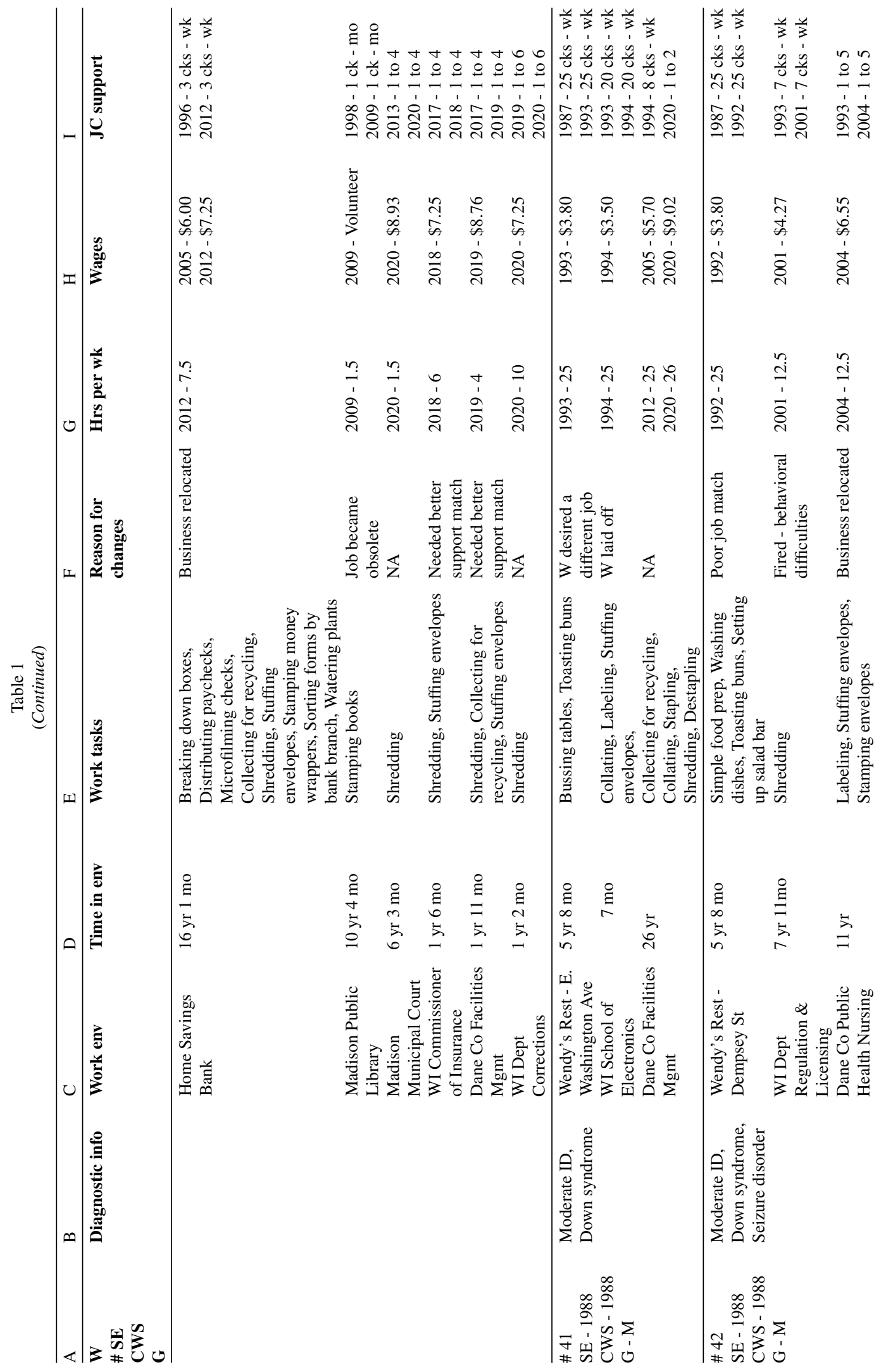




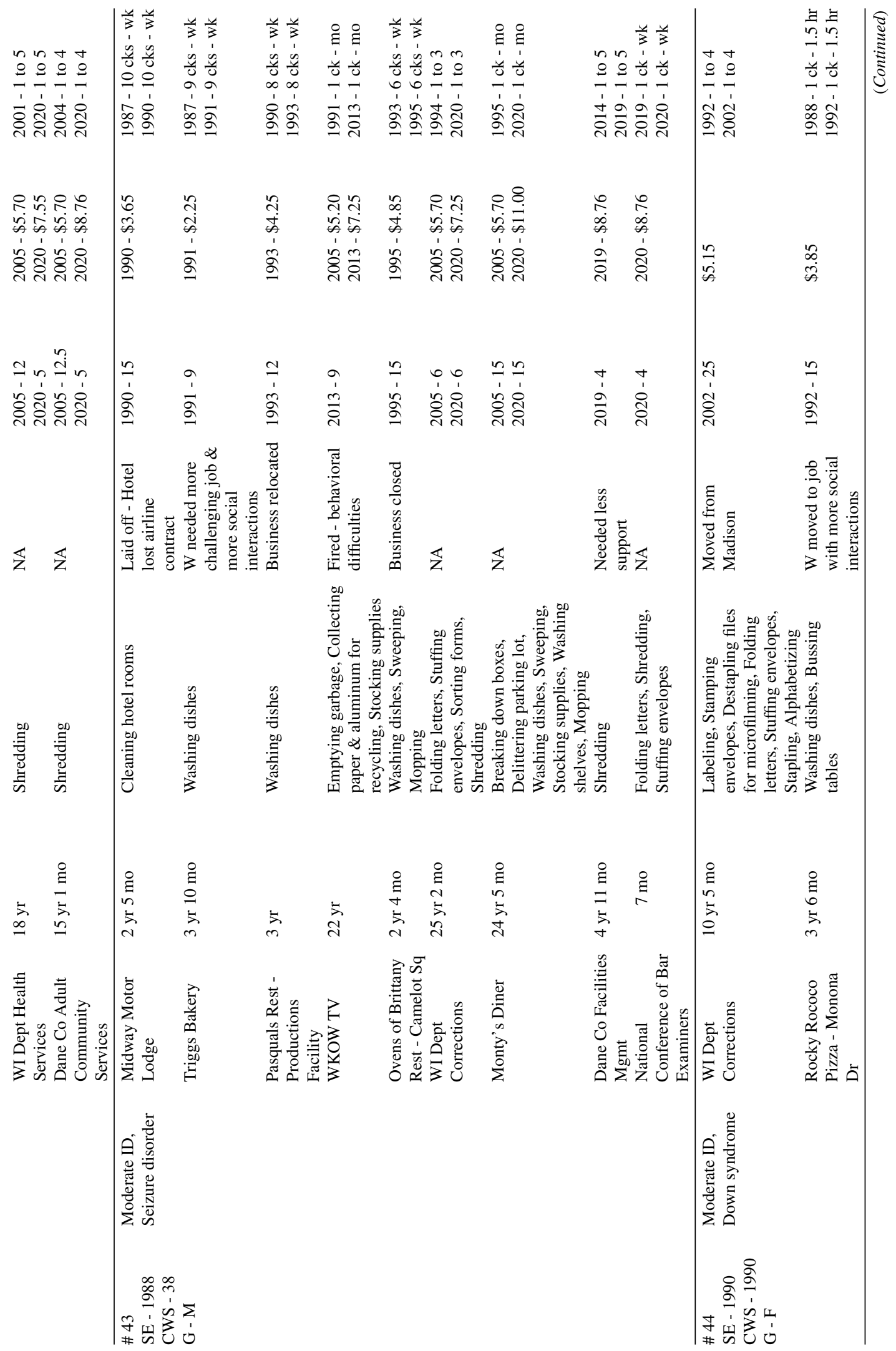




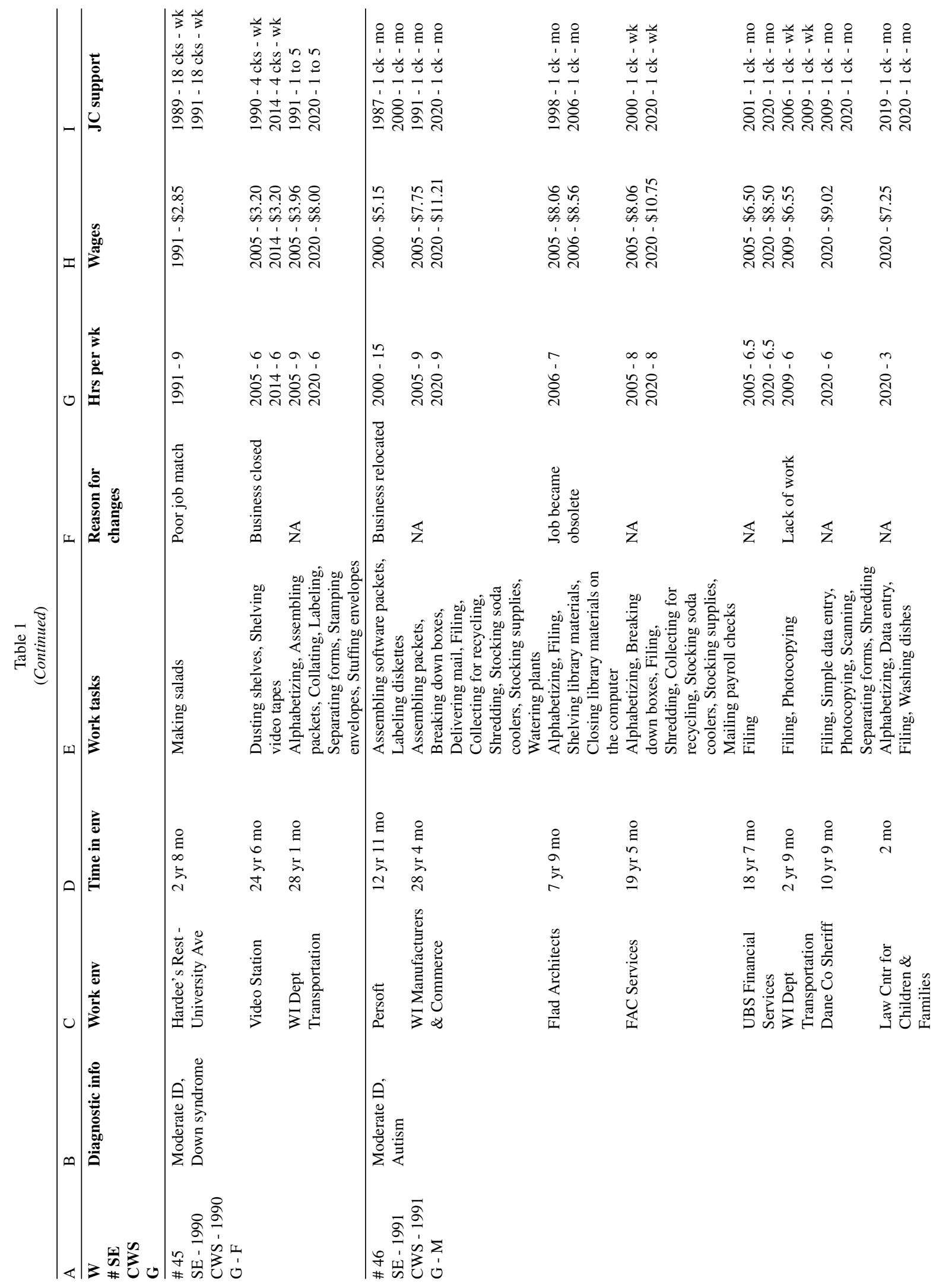




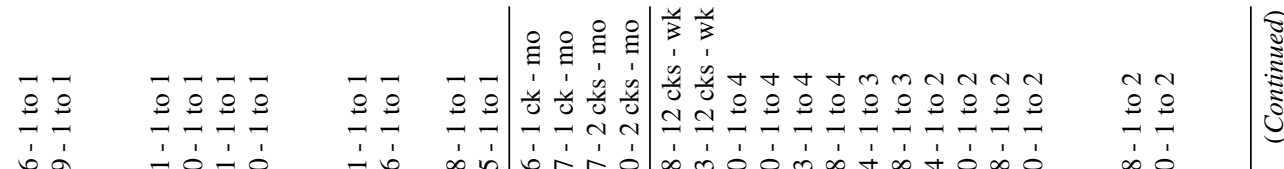

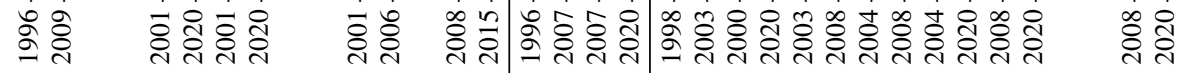

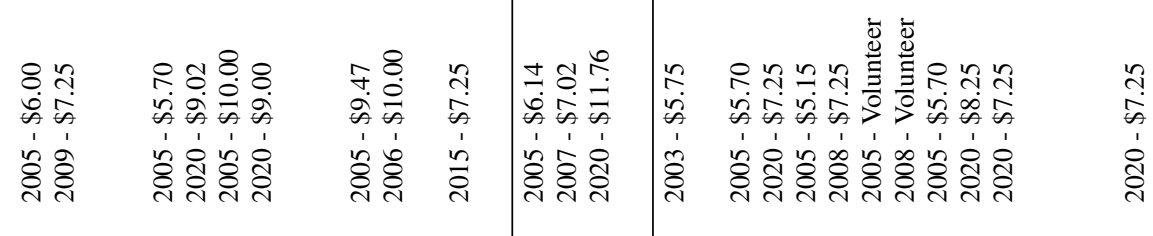

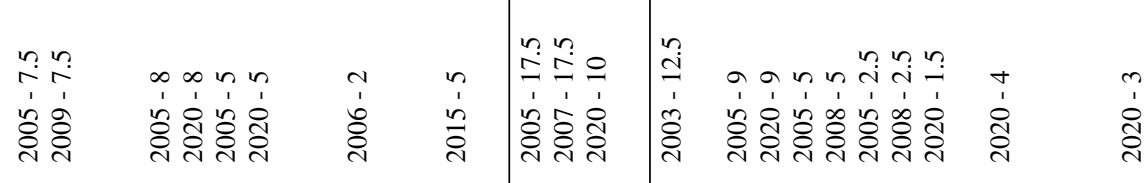

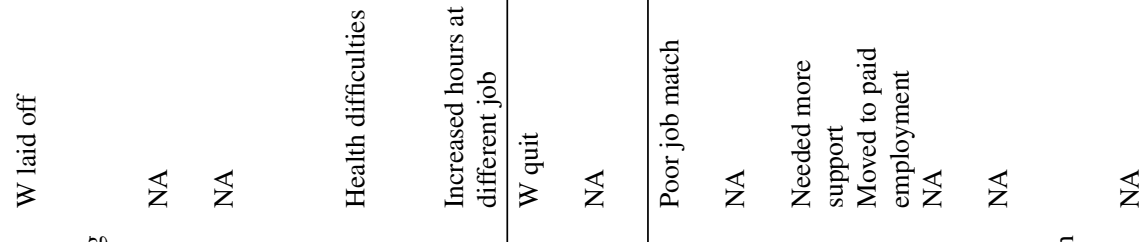

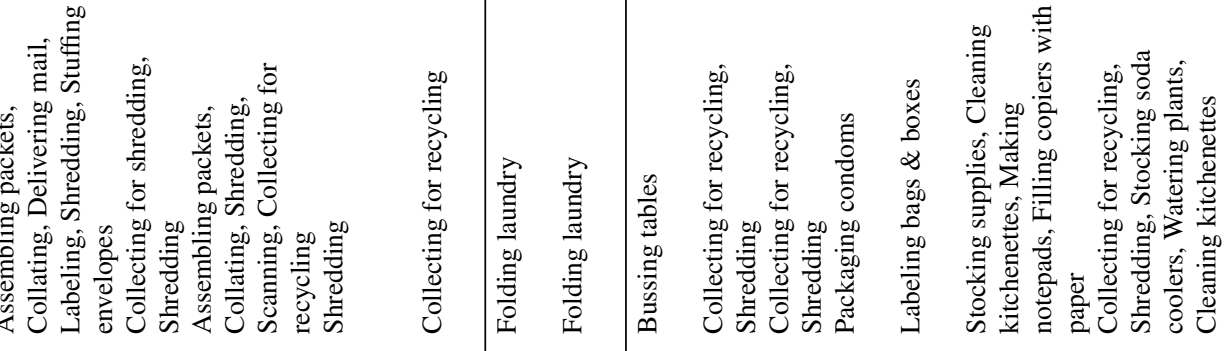

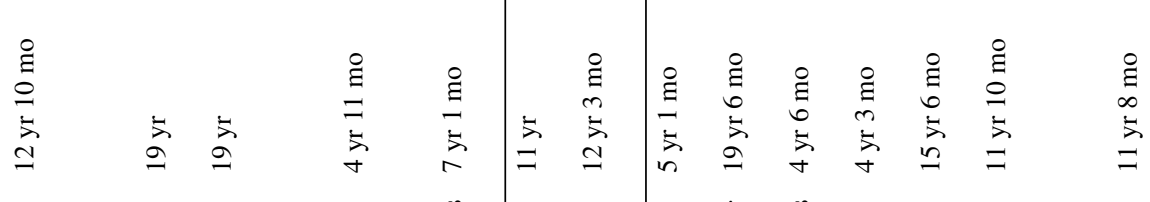

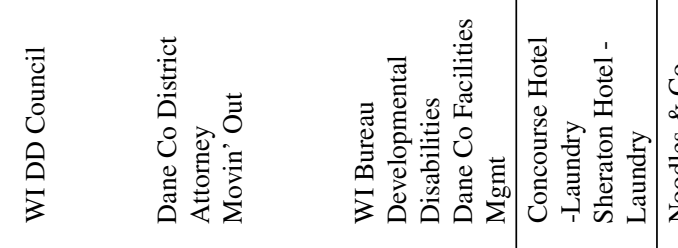

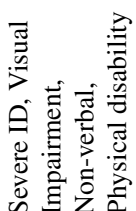

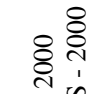

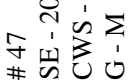

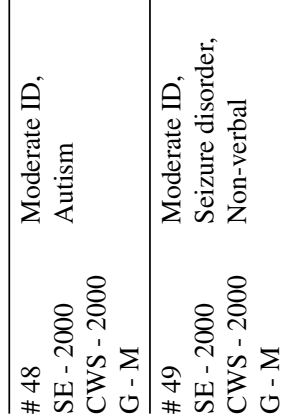




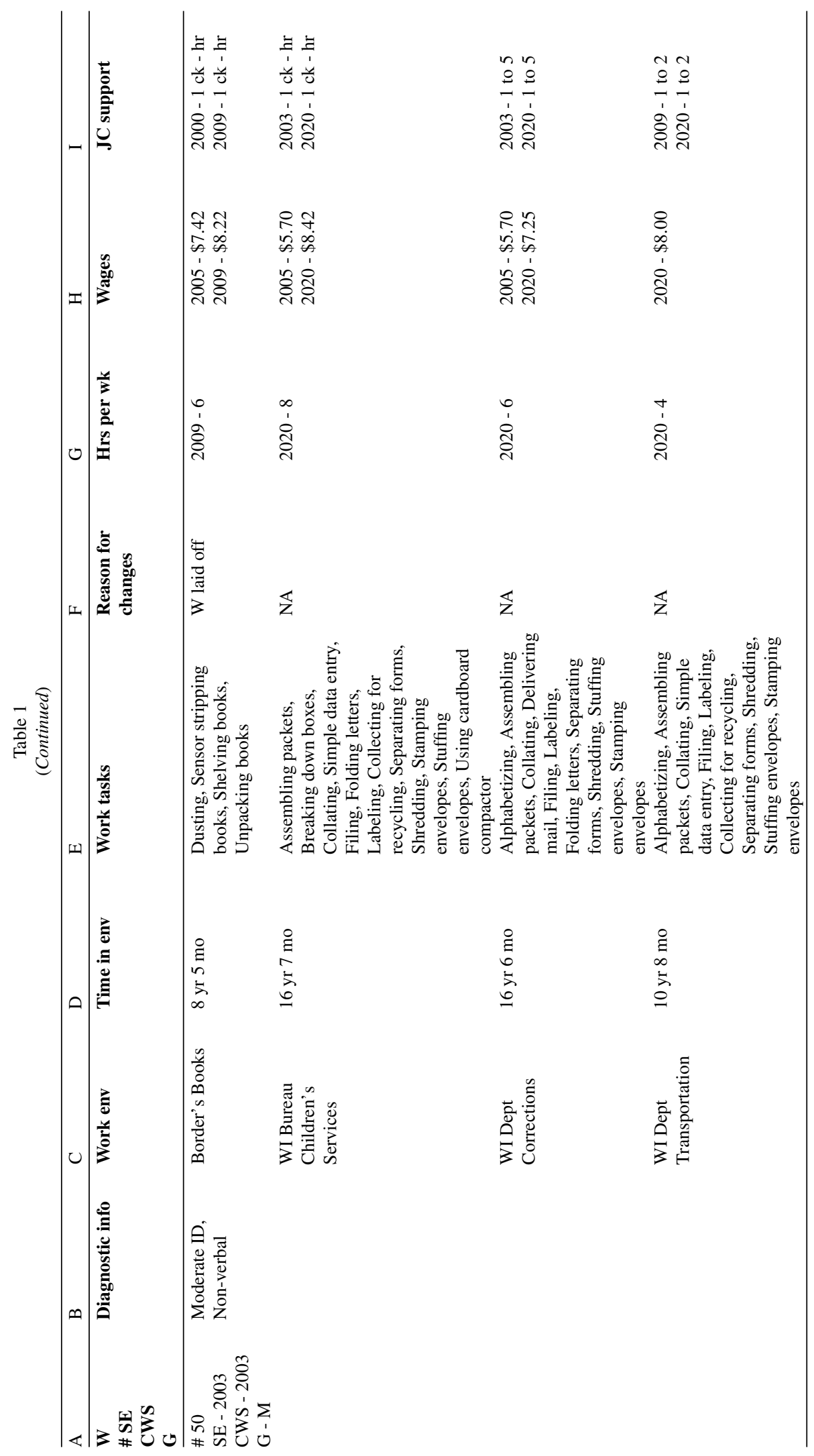




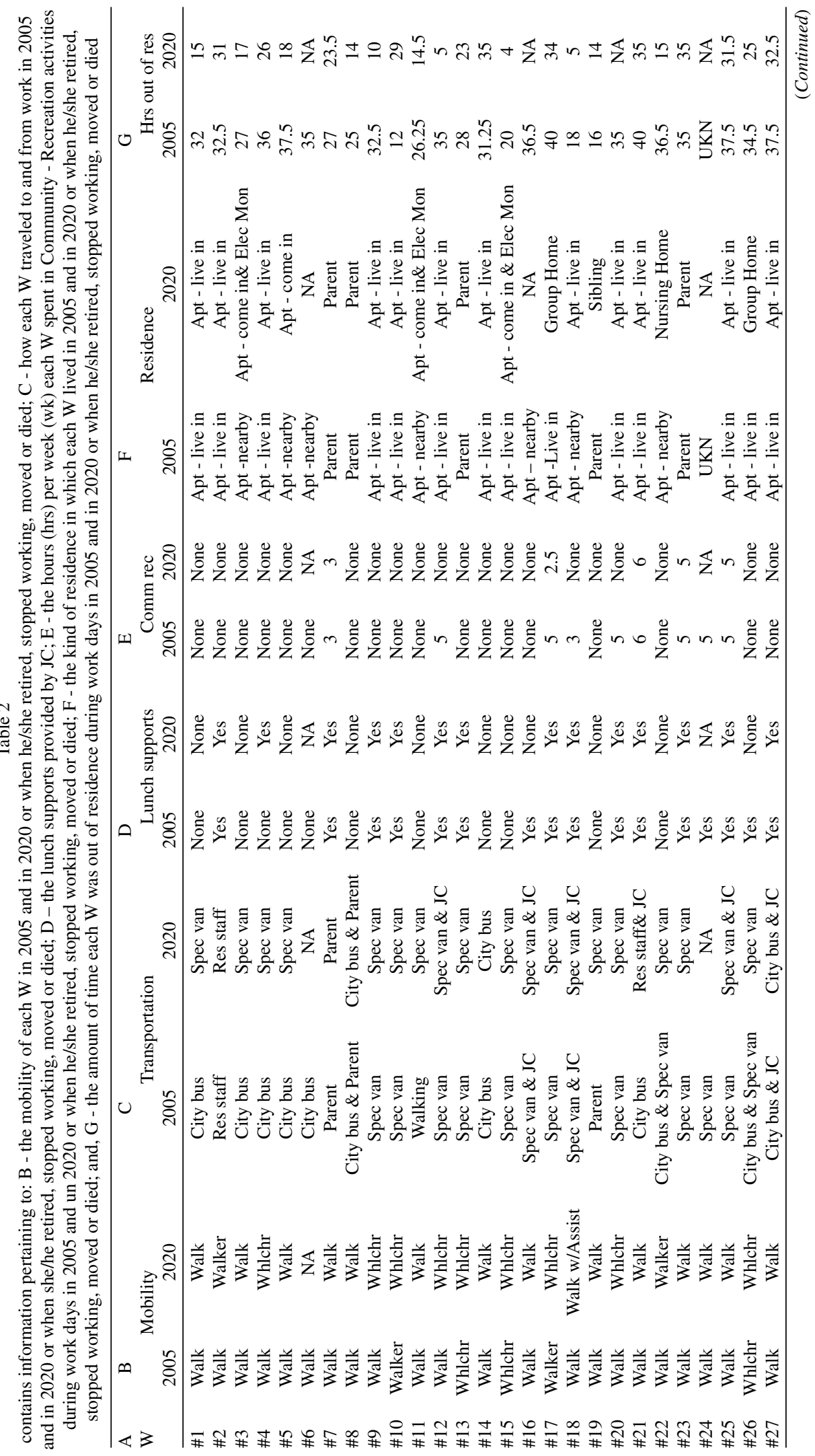




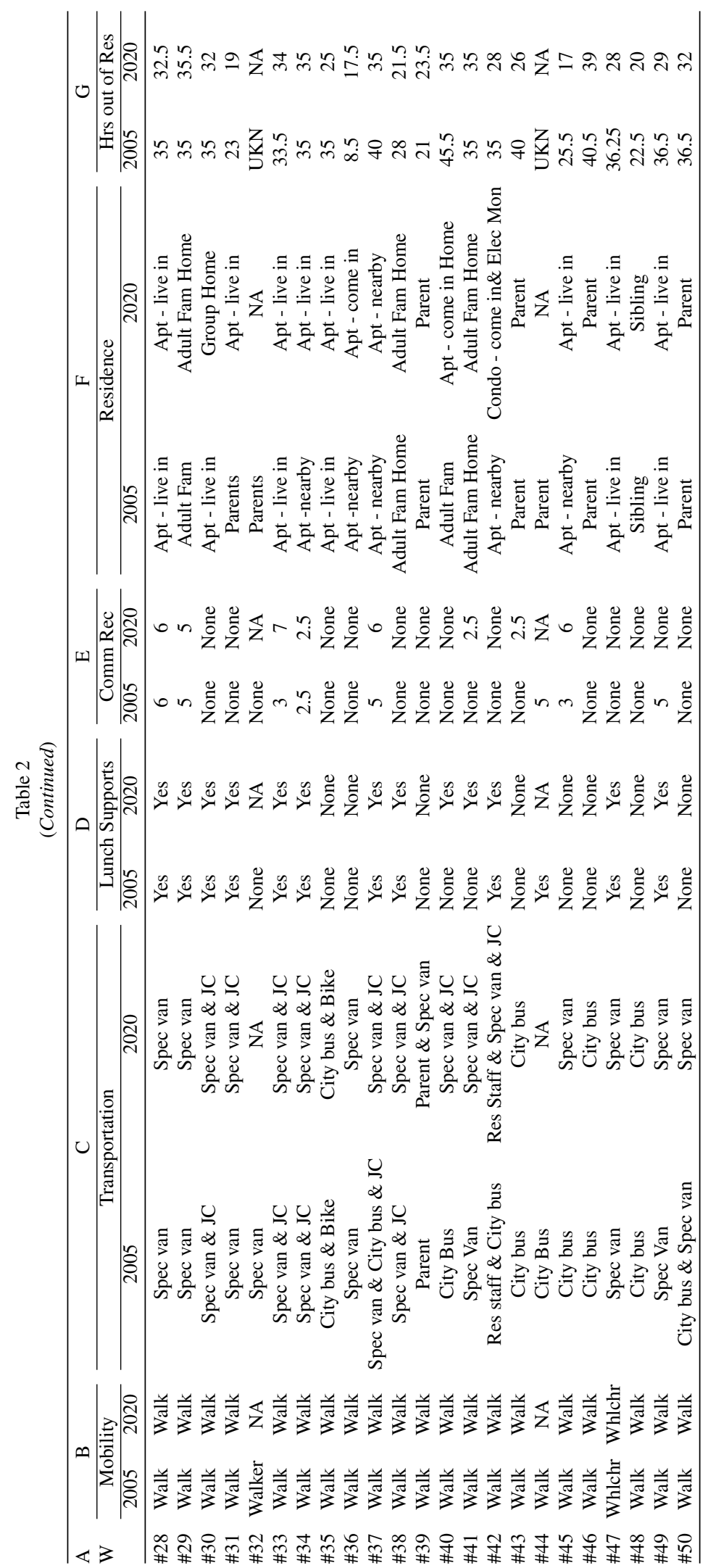


"moderate" intellectual disabilities; nine as having "severe" intellectual disabilities; and one, who had significant cerebral palsy, was "estimated" to have "mild" intellectual disabilities. Twenty one had Down syndrome, ten of those were female. Thirteen were nonverbal, four had seizure disorders, five had hearing impairments, five had vision impairments, five had long term significant physical disabilities, seven had Cerebral Palsy, five had Alzheimer disease and two had significant Arthritis. Seven others had one of the following: Diabetes, Dementia, Schizophrenia, Cornelia de Lange syndrome, Microcephaly, Heart Disease or Prader-Willi Syndrome.

All 50 functioned intellectually and otherwise among the lowest approximately $1 \%$ of a naturally distributed general population. All experienced communication, intellectual, social, physical, behavioral, sensorimotor and/or other disabilities in kinds and degrees reasonable persons would consider significantly disabling. All needed substantial long term assistance in almost all activities of daily living. All but one had been ascribed standardized, adapted and/or "estimated" intelligence test scores of approximately 50 or below. While in school none of the workers performed well, if at all, on standardized tests of any kind. None was successful in academic curricula. All looked or behaved in ways that called immediate attention to their difficulties. All were judged eligible for long term vocational support by Dane County officials and all were eligible for Social Security Disability Insurance (SSDI) and/or Supplemental Security Income (SSI). Other than family members few others interacted with them. Thus, it was relatively easy to think they could not learn or do much. As will be obvious from the data that follow, those who thought so were wrong.

\subsection{Integrated work environments}

When the possibility of integrated work was first proposed, most who were aware of the physical, intellectual and behavioral characteristics of the students and the histories of their predecessors were incredulous. Where could THEY work? Who would hire THEM? TERRIBLE things will happen to them. They will do TERRIBLE things there. Thus, it was incumbent on those who proposed the possibility to generate the needed environments. Many of the integrated work site development strategies utilized are reported in Brown \& Kessler (2014). The policies and practices of CWS are to constantly generate new and different integrated work environments because workers leave, businesses move, close or reduce work forces and many jobs become obsolete due to technological advances.

A large integrated work environment has three major characteristics. First, it is naturally proportioned. That is, no more than one or two of those who work in it should be significantly disabled intellectually because no more than $1 \%$ of the general population can be so described. Consider a bank that employs 100 individuals. To be naturally proportioned, no more than one or two of those who work therein can be significantly disabled intellectually. Second, no more than two individuals with obvious disabilities can work at the same time in an immediate work environment. Third, a worker with significant intellectual disabilities must work within sight, sound and touch of coworkers without disabilities. This is to facilitate appropriate natural supervision, the development of social relationships and safety. Historically, all the environments in which CWS workers functioned met these criteria. Severe budget reductions forced the utilization of three settings that contained more than two workers each.

Table 1 - Column C contains a listing of the integrated work environments in which the 50 workers functioned from school exit to 2020 or until they died, retired, stopped working or left Madison. Workers \#1 through \#7 functioned in integrated work environments prior to school exit and were maintained therein by other vocational agencies until CWS started providing them support services in 1984.

When a work environment is considered only once, the 50 workers functioned in over 160. In many instances, more than one worker functioned in a particular environment. The names of some environments changed over time. In such instances, the most recent name was used for all.

There were substantial changes in the kinds of environments utilized over time. In 1986, 20 food service and 22 office environments were utilized by the 40 workers served at that time. In 2006, the same 40 workers functioned in only six food service settings, but in 47 office environments. In 2020, the 36 remaining workers functioned in 53 office and only four food service environments. Several workers functioned in more than one office setting. More office environments were utilized for several important reasons. First, offices often have lower turnover rates than those of food service settings. This allows for the frequent contacts and common experiences over 
extended periods of time which are so necessary for the development of meaningful social and supportive relationships with coworkers without disabilities. Second, some parents preferred office environments because they perceived them as having higher status and as being more intellectually challenging. Third, many office tasks are less physically and otherwise demanding than those required in food service environments. Fourth, Madison contains an unusually large number of city, county, state, federal, university and private sector offices. Fifth, public busses and the central locations, accessibility and the close proximity of many office buildings allowed for the efficient movement and stationing of workers and Job Coaches.

Many workers functioned in two or more work environments in a day or week, primarily for the following reasons. At times one environment did not provide the variety of tasks that would maintain interest, willingness to perform and physical well-being. Multiple environments provided more opportunities for the preferred amounts and kinds of social relationships with coworkers without disabilities. A reasonable balance between active and sedentary tasks across a day or week sometimes was not available in one setting. There was an insufficient amount of work in one setting. Workers who presented significant social and behavioral challenges were often more readily accepted or tolerated if they functioned in a setting less than a full day or week. If a worker functioned in two settings and lost access to one, it was usually less disruptive and easier to arrange another part time rather than a full time setting. A setting may have required skills that were in the repertoire of a worker during some, but not all, parts of the work day or week.

The amount of time each worker functioned in each environment is presented in Table 1 - Column D. The total amount of time the 50 workers spent in integrated work environments was over 579 years. Individual amounts of time ranged from two months to 42 years. The average amount of time spent in a work environment was 11.5 years.

The "Where can THEY work?" and the "Who would hire THEM?" questions have been answered. They can and in fact do work in banks, hospitals, restaurants, research laboratories, radio stations, fire departments, police departments, crime laboratories, offices, taxi cab companies, law firms, architect companies, construction companies and in many other integrated environments. Those who predicted employers would not open their doors to workers with significant intellectual disabilities were wrong. The over 100 employers reported here and at least 100 others in Dane County, WI who did so are the evidence. Also, the "They will do TERRIBLE things there" and "TERRIBLE things will happen to them" predictions have not been realized. Not one worker was sexually or otherwise abused. Not one sexually or otherwise abused others. Not one was arrested. Not one became pregnant. Not one has been injured seriously at work. Not one has been lost or otherwise harmed while under the supervision of CWS personnel.

\subsection{Work tasks}

In the 1970's when permission to bring the students to integrated work sites was requested from parents and school officials, many were astounded and asked "What can THEY do there?" Thus, it was incumbent upon those who generated the sites to determine the real work tasks that might be learned and performed. Many of the strategies utilized to do so are reported in Brown, Kessler, \& Toson (2016).

A "real job" refers to the entire range of work tasks a worker without disabilities must perform acceptably in order for an employer to hire or continue to employ her/him at least the minimum wage. Many work tasks required of coworkers without disabilities were too difficult for the workers. Answering telephones, using computers, reading, understanding complex language and verbally assisting customers are examples. In addition, many workers manifested attendance rates and/or physical and behavioral difficulties and response rates that would have been tolerated by few employers, if they were not disabled. In short, none of the 50 workers could successfully complete all the work tasks required of any coworker without disabilities. However, each worker could perform one or more of the work tasks of any coworker without disabilities. If a worker with disabilities does not complete a work task in accordance with the minimally acceptable standards of an employer, and as a consequence a coworker without disabilities must be paid to do so, it is considered a real work task. No business can succeed if it is dependent only upon the productivity of workers with the kinds and degrees of disabilities operative here. With the possible exception of a few volunteer experiences, all workers performed real work tasks in accordance with this definition.

In some instances, a worker could complete some tasks in the same manner as coworkers who were not 
disabled. In others, she/he could do so only if individualized adaptations were utilized. An adaptation refers to something that is added to or changed in the setting that allows a worker with disabilities to function in accordance with the minimally acceptable standards of an employer. These adaptations are not needed by coworkers without disabilities. Some adaptations such as paper folding devices, color coded mail folders and pictures of performance sequences are relatively simple and inexpensive. Others are quite complex and costly. Opportunities to learn and perform a wide array of different and more or less complex tasks were continuously available. From 1984 to 2020, the 50 workers performed well over 150 real work tasks which are delineated in Table 1 - Column E.

The question "What can THEY do there?" has been answered. Workers with significant intellectual disabilities can and are successfully completing hundreds of real work tasks. Those they perform allow their more able coworkers to complete more complex and valuable tasks.

\subsection{Reasons for changes in environments and tasks}

The number of work environments in which an individual worker functioned ranged from 1 to 12 . The average was six. The major reasons for changes in environments and tasks are presented in Table 1 Column F. Changes were made primarily to enhance opportunities for and to engender better job matches. For example, changes were made because the array of tasks was or became too limited, complex or simple. In some instances, an environment and its requirements were a good match at one point, but became less so over time. Deteriorating physical and intellectual functioning were most often the reasons. At times, a different environment offered better opportunities for social interactions with coworkers without disabilities and more natural supports. Natural support refers to assistance provided by coworkers without disabilities that does not interfere with their productivity or the enjoyment of their jobs. To move a worker from nonpaid to paid employment or to use a site closer to a residence so as to reduce travel time, cost and support requirements and when a worker became disinterested in an environment or tasks or otherwise indicated discontent required changes. "Indicating" a change is wanted took different forms, but was rarely verbal. Obvious declines in performance, exhibiting disruptive behav- iors and refusing to go to work are examples. When a worker was fired, laid off or otherwise terminated, the major reasons were unacceptable social or behavioral actions, excessive absenteeism and poor work quality. Social or behavioral actions that warranted dismissal included mild aggression toward others, inappropriate demands for attention that interfered with the functioning of coworkers and supervisors and stealing. Pens, coffee cups, post it notes and snack foods were the items taken most frequently. Money was rarely taken, but was not tolerated when it was. In most instances a worker was not terminated until several problematic actions were expressed. Finally, if a Job Coach sensed that coworkers, supervisors or employers were becoming increasingly uncomfortable, preemptive changes were often made.

Layoffs resulted from declines in business, work tasks being phased out or becoming obsolete, work being assumed by fulltime employees and there being an insufficient amount of work available. From 1984 to 2020, 36 years, CWS never moved a worker from an integrated to a segregated work environment. When changes were necessary, they were moved to other integrated environments after short periods of time.

\subsection{Hours worked per week}

The hours each worker worked per week are presented in Table 1 - Column G. Generally, workers were the responsibility of CWS from 8:00 A.M. to 3:00 PM, Mondays through Fridays. This encompasses time spent working, in lunches, traveling to and from workplaces and participation in Community and Recreation activities. In 2005, the number of hours per week 50 workers spent working ranged from six to 30.5. The average was 20.15. In 2020, the number of hours per week the 46 workers spent working ranged from eight to 26 . The average was 14. From 1984 to 2020, 18 worked about the same number of hours per week, seven worked more and 25 worked fewer. This is noteworthy because as the workers aged, stamina and more serious intellectual, physical care, mobility and other difficulties quite often reduced functioning levels and required that more time and energy be devoted to non work activities. The major reasons for changes in hours worked per week over time are as follows. Employers requested fewer hours, primarily because of the lack of available work. Workers, parents or support teams desired more or fewer hours. Changes were made so CWS could provide needed supports. Health, intel- 
lectual and/or behavioral problems often led to fewer hours. There was or became a less than acceptable match between a worker and an environment. The worker seemed uninterested or otherwise communicated a choice for a new task or environment. The task became obsolete, primarily because of technological innovations.

\subsection{Wages}

Wages earned from 1984 to 2020 are presented in Table 1 - Column H. In 2005, the WI legal minimum hourly wage for private sector employees was $\$ 5.70$. The prevailing city, county and state wages per hour in the Madison area for entry level positions was approximately $\$ 9.00$. The hourly wage of the workers in, or prior to, 2005 , ranged from $\$ 3.20$ to $\$ 10.00$. The average was $\$ 5.76$. In 2020, the WI legal minimum hourly wage for private sector employees was $\$ 7.25$. The prevailing city, county and state hourly wage in the Madison area for entry level positions was approximately $\$ 11.00$. The hourly wage range of 46 workers from 2006 to 2020 was from $\$ 6.00$ to $\$ 11.76$. The average wage per hour was $\$ 7.96$.

None of the workers ever worked 40 hours per week. Because of the part time nature of their work, only a few received medical or other benefits through employers. No worker could live on the wages she/he earned. The ranges and amounts of work produced were almost always less than those of coworkers without disabilities. The productivity of all workers was documented empirically by CWS personnel. Some employers were eligible for deviated wage certificates that allowed subminimum wages commensurate with productivity. However, from 2017 to 2020, no worker was paid a subminimum wage.

Only one of the 50 workers made about the same total amount of money over time. Eleven made less total amount of money over time. Thirty eight made more total amount of money over time. Increases in the legal minimum and prevailing wages accounted for most of the increases in the total amounts of monies earned over time. However, some workers made more money per hour, but worked fewer hours. Thus, they earned less total money. As the workers aged, many had to reduce the hours they worked because of health, stamina and related conditions and their incomes decreased.

A worker was considered a nonpaid volunteer if she/he or his/her support team had reasonable knowledge about, and agreed to perform, the required tasks and if the setting was legally used by others without disabilities as volunteers. In 2005, seven workers functioned as volunteers. Each also had paid employment in other settings. From 2006 to 2020 or until a worker died, retired, stopped working or left Madison, 18 functioned as volunteers. In 2020, only six did so. They also had paid employment in other settings.

Volunteer arrangements were utilized primarily for the following reasons. First, the physical, social, logistical and other benefits of volunteering were deemed more important than the amounts of money that could be earned elsewhere. Second, as the competence and productivity of some workers increased, so did their earnings. CWS personnel and parents/guardians took the steps necessary to make sure that wages did not negatively impact SSI/SSDI benefits. Third, some workers or their support teams realized more money could be earned elsewhere, but clearly preferred for personal fulfillment reasons to function as volunteers. A worker who loved music clearly preferred to volunteer at a radio station and a worker who loved to visit family members and friends at a hospital and clearly preferred to function therein as a volunteer are examples. Fourth, it was important that workers function outside their residences reasonable amounts of time per week. Sometimes the only or the best way to generate out of residence time was to arrange for volunteer experiences. Fifth, volunteer arrangements were used in emergencies. In cases of health crises and recoveries, terminations and layoffs are examples. Sixth, volunteer experiences offer good opportunities to learn important skills and attitudes. These opportunities sometimes led to paid employment. For example, one worker functioned in a fire station as a volunteer. After he learned to complete work tasks successfully, the firefighters utilized monies from their charitable trust to pay him.

\subsection{Job coach support}

Extra support refers to the amounts and kinds of assistance a Job Coach provides that would not be needed if a worker was not disabled. None of the workers could have been successful in a workplace without the comprehensive and sustained extra assistance of Job Coaches. The amounts of support provided individual workers are reported in Table 1 - Column I. They ranged from onsite spot checks of approximately 15 minutes to continuous supervision as long as a worker functioned in a workplace. 
Most of the support provided by Job Coaches included the following.

- Providing direct and systematic instruction.

- Verifying that work tasks were completed acceptably and that negotiated routines and schedules were followed.

- Motivating and encouraging workers.

- Assisting coworkers, employers and supervisors setting up workers with assigned tasks.

- Monitoring behavioral challenges and following through with agreed upon interventions.

- Monitoring dress and grooming requirements.

- Assisting with personal care.

- Programming and improving augmentative communication devices.

- Monitoring, evaluating and improving the effectiveness of individualized adaptations.

- Setting timers and alarms that indicated work breaks and moves to another worksite.

- Gathering evaluative information from employers and coworkers about worker performance and responding constructively.

- Assisting during transitions to and from worksites, lunchrooms and transportation points.

- Responding appropriately to extraordinary situations such as medical and behavioral emergencies.

Job Coaches were taught and encouraged to be sociable, accessible and approachable. They were also encouraged to provide non confidential but relevant information about a worker's interests, abilities and communication styles as ways of facilitating and increasing opportunities for social interactions. When Job Coaches initiated social contacts on behalf of a worker, coworkers were generally happy to be approached. Often, they did not realize that becoming involved with a worker in social activities within or outside the workplace was appropriate and preferred. After initial contacts and connections were made by Job Coaches, coworkers often continued and expanded them with minimal support. In a few instances meaningful social relationships developed quickly and with relative ease. However, it was quite common for them to develop gradually. One particularly helpful maneuver was for Job Coaches to model desired social interactions. For example, for two years a Job Coach brought cake and gifts to the office to celebrate the birthday of a worker. In the third year, coworkers organized his birthday party without the involvement of the Job Coach.

As many wonderful social relationships developed, it became apparent that the workers seemed to be receiving much more than they were giving. Thus, with the assistance of Job Coaches, workers began acknowledging the birthdays of coworkers and employers, inquiring about vacations, sending get well cards and making other gestures common in reciprocal relationships. After initiating more "giving" actions, the workers became even more socially connected to coworkers and employers. Oftentimes the excitement and novelty of work settings and tasks fade. It was quite common that social relationships with coworkers produced enduring satisfaction and sustained productivity.

In 2020 CWS served 79 workers with the equivalent of 17 full time Job Coaches. From a budgetary perspective, this could be interpreted as a support ratio of approximately 1 to 4.6. However, from a program operations perspective, this is not an appropriate conceptualization. Specifically, in some instances a Job Coach was responsible for a fixed number of workers. However, illness, injuries, training, vacations and other factors resulted in her/him being unavailable. The Executive Director, the Program Director and other Job Coaches then provided the needed support. Job Coaches continuously strived for safe, cost efficient and otherwise individually meaningful balances between too much and too little support. Some workers functioned quite well in high ratio support arrangements because of their personal care, social, behavioral, travel and work repertoires. Others functioned quite well in high ratio arrangements during work times, but needed lower ratio arrangements during relatively unstructured lunches, when traveling and in Community and Recreation activities because of safety concerns, mobility problems, personal care needs and behavioral challenges. If work tasks were within the lower end of the difficulty range of a worker and were being performed acceptably, the support required to ensure acceptable work quality was minimal. When a worker was learning new or more complex tasks, increases in support were always necessary. One day a worker could have been functioning quite well with weekly or monthly spot checks. However, the next day, for health, behavioral, instructional or other important reasons, she/he may have needed extra support for the entire time she/he was working. As the workers aged, deteriorating mental, physical, health, personal care, rate range and other skills required increasing amounts and kinds of support. A worker may have functioned quite well with only a few spot checks in a particular environment mornings. This allowed a Job Coach to serve others in other environments 
and thus increases the support ratio. However, the same worker may have functioned in a different environment afternoons with another worker who needed substantially more support. This lowered the support ratio. In some instances, budget reductions affected worker pairings. For example, one worker was functioning quite well with weekly spot checks. Another worker was having serious difficulties in another setting and needed constant support. CWS considered many factors and decided to pair the two. In sum, professional support had to be individualized, flexible and responsive to constantly changing circumstances. In Table 1 - Column I the support arrangements are reported. They are categorized as follows.

- 1 to 1 or 1 to 2 . In these arrangements a Job Coach was in the same physical space, in sight of and/or in close proximity to one or two workers almost $100 \%$ of the time. Constant and close presence was deemed necessary to: maintain concentration on tasks and productivity; address seizure disorders and other health concerns; honor court orders; address strong parent preferences; manage behavioral challenges; minimize interference with the productivity and enjoyment of others; and, attenuate safety risks.

- 1 to 3, 4, 5, or 6. In these arrangements Job Coaches were approximately five minutes away from the three to six workers for whom they were responsible. For these arrangements to be operative and yet to honor the criteria of an integrated work environment, settings that were in relatively close temporal and spatial proximity were used. For example, five workers functioned in four separate areas of a large government office building. This allowed a Job Coach to check each every 15 to 20 minutes. When necessary, supervisors and coworkers without disabilities used cell phones and pagers to contact Job Coaches who were rarely more than five minutes away.

- Daily checks. In this arrangement Job Coaches typically checked workers at least once per day. However, depending on support needs, a worker was checked once in 30 minutes to once in four hours. The typical routine of a Job Coach was to rotate across workers throughout a morning or afternoon. Because some workers functioned acceptably with less assistance than others, their work environments were more dispersed. However, a Job Coach was rarely more than 15 to 20 minutes away.
- Weekly and monthly spot checks. In this arrangement workers were checked by a Job Coach one to three times per week or month. Typically, a check lasted from five to 30 minutes.

Remarkably, the kinds and amounts of Job Coach Supports provided 25 of the 50 workers remained about the same over their careers. Only three of the 50 workers had decreased extra supports provided over their careers. As the workers aged, health and related difficulties took their toll. Thus, the extra supports provided 22 of the 50 workers increased over time. These increases, of course, lowered support ratios and increased budgetary difficulties.

\subsection{Mobility}

How the 50 workers ambulated in 2020 or when they died, moved from Madison, retired or stopped working is reported in Table 2 - Column B. Before 2005,43 of the 50 workers walked without the personal assistance of others, three used walkers and four used wheelchairs. By 2020, 33 of the remaining 46 walked. One walked, but only with the personal assistance of others, two used walkers and ten used wheelchairs. These declines in mobility skills had important, pervasive and in some cases dramatic effects on virtually all aspects of daily living. Of particular importance is they often decreased time working, increased time in residences and increased time not working but out of residences. They also lowered worker to Job Coach ratios and thus increased costs. Obviously, the decreases in mobility skills reported here are no different than those experienced by individuals without significant intellectual disabilities. However, individuals without disabilities typically experience the kinds and degrees of difficulties reported here 15 to 20 years later in life.

\subsection{Transportation}

How the 50 workers traveled to and from work from 1984 to 2005 or when they died or left Madison is reported in Table 2 - Column C. Specifically, from 1984 to 2005, 13 used city busses, 18 used special vans, six used special vans and Job Coaches, three used city busses and special vans and three used parents. One each walked, used city busses and Job Coaches, city busses and parents, residence personnel, city busses and a bicycle, residence personnel and city busses, and Job Coaches, special vans and city busses. 
From 2006 to 2020, 22 of 46 workers traveled to and from work in special vans, 12 were transported by Job Coaches and special vans and four used city busses. One each was transported by parents, parents and special vans, parents and city busses, city busses and Job Coaches, residence personnel, city busses and a bicycle, residence personnel, city busses and Job Coaches, and special vans and city busses.

As the workers aged, major changes in transportation to and from work were necessary. In 2005, 13 of the workers used city busses exclusively. In 2020 only four did so. In 2005, 18 workers used special vans exclusively. In 2020, 22 did so. In 2005, six workers used special vans and Job Coaches. In 2020, 12 did so. In 2005 three parents transported their children to and from work. In 2020 only one did so. The major reasons for using other than typical transportation modes were: safety, mobility difficulties, weather, accessibility, health and seizure disorders.

The changes in transportation modes required more time from Job Coaches and CWS leadership personnel and substantial tolerance, understanding and cooperation from parents, guardians, coworkers, employers and residential personnel. They also forced reductions in worker to Job Coach ratios and thus increased costs.

\subsection{Lunch}

Lunch time was from approximately 11:30 AM to 12:30 PM. Lunch times were often longer than those of coworkers without disabilities because more time was needed for mobility, purchasing food, cutting up food, opening packaged items, drinking, eating, personal care and cleaning up (Table 2 - Column D). Some lunched with coworkers without disabilities and some did so with peers with disabilities and/or Job Coaches. Some workers needed little or no extra support during lunches throughout their careers. However, many required increasing kinds and amounts of extra support as time passed.

Between 2006 and 2020 or when a worker retired, died, stopped working or left Madison, more workers needed assistance and more needed more significant assistance. Some expressed behavioral difficulties in relatively unstructured lunch periods. In such instances a Job Coach ate with from two to five workers in the same location. This was an unfortunate violation of natural proportions. It was done because the personnel needed to supervise low ratio lunch arrangements could not be afforded.

\subsection{Community and recreation activities}

Between 1984 and 2005, or before two workers died and two left Madison, 16 of the 50 workers spent from 2.5 to six hours per week in Community and Recreation activities during work days and times with a Job Coach in groups of two or three (Table 2 Column E). The average was 4.5 hours per week. The activities in which they engaged included swimming, shopping, using public libraries, going to the zoo and museums, visiting a bakery, playing mini golf and bowling.

Between 2006 and 2020 or before they died, retired, stopped working or left Madison, 14 of the remaining 46 workers engaged in Community and Recreation activities during portions of their work weeks. The hours per week ranged from 2.5 to seven. The average was four.

The workers engaged in Community and Recreation activities during work days and times for several reasons. It was important that most workers be out of their residences between approximately 8:00 A.M. and 3:00 PM. In some instances, appropriate work experiences could not be arranged for the needed amounts of time. Behavioral difficulties and limited stamina disallowed full days at work. In some lives, Community and Recreation experiences during non work days and times were too few or nonexistent. Support teams judged that involvement in fun and healthy alternatives were needed. Weight, stamina and fitness difficulties encouraged engagement in generally healthy Community and Recreation activities. In a few instances Community and Recreation activities were used as incentives; e g., "When I work, I get money to shop, eat at a restaurant."

\subsection{Residences}

Table 2 - Column F contains information about the type of residences from 1984 to 2005 and from 2006 to 2020 or when a worker died, stopped working, retired or left Madison.

None of the 50 workers was intellectually, physically or otherwise capable of living "independently". That is, without comprehensive extra support provided by individuals without disabilities. Residential support services included, but were not limited to, providing assistance in the areas of hygiene, food purchasing and meal preparation, dressing, laundering, personal maintenance, traveling and medication, schedule and money management. 
From 1984 to 2005 or when a worker died or left Madison, 20 of the 50 lived in apartments with live - in support. That is, they lived with no more than one other person with disabilities and a person without disabilities who was paid to live with them. Twelve lived in apartments with nearby support. An apartment with nearby support refers to one in which one or two individuals with disabilities reside. Support services are provided by paid persons without disabilities who lived close to the apartments. In some instances, those persons lived in apartments in the same buildings as the workers. Twelve lived with their parents. Four lived in three different adult family homes. An adult family home is essentially a foster home for adults. One lived with a sibling. It was unknown where one worker lived. From 2006 to 2020 or when a worker died, retired, left Madison or stopped working, 20 of the 50 lived in apartments with live - in support, one lived in an apartment with nearby support and three lived in apartments with come - in support. An apartment with come - in support refers to one in which one or two individuals with disabilities reside. Support services are provided by paid persons without disabilities as needed. Those who provide the services do not necessarily live near the apartment. Three lived in apartments with come in support plus electronic monitoring. This monitoring included, but was not limited to, alarms on doors and bedside floor mats which detected movements, emergencies and other potentially dangerous situations. One lived in his/her condominium with come - in support plus electronic monitoring. Eight lived with parents. Three lived in two adult family homes. Two lived with siblings. Three lived in group homes. A group home refers to an apartment or house with more than two unrelated persons with disabilities. One lived in a nursing home. Two of the 50 moved from Madison prior to 2005 and one moved between 2005 and 2020. Their residential status is unknown.

The average number of hours per week 46 of the 50 workers spent out of residences during work days and times in 2005 or when they died or left Madison was 32.2 (Table 2 - Column G). The average number of hours per week 45 of the 50 workers spent out of residences during work days and times in 2020 or when they died, retired, stopped working or left Madison was 25. The amount of time five workers spent out of residence is unknown. In many instances, the workers spent fewer hours per day out of their residences as they aged. The major reasons for this were stamina, illness and the choice of a worker, a parent and/or a support team.

\subsection{Familial succession/mortality}

Two people produce a child and thus become parents. Culturally, biologically and otherwise they have responsibilities for the wellbeing of their offspring. Their child then becomes a parent. She has responsibilities for both her children and her parents, who are now grandparents. The generally expected order of familial succession is that grandparents will die first, followed by parents and then by their children. War, pestilence, famine, accidents, drought, sexual preferences, etc. have always been departures from this order. Individuals with significant intellectual disabilities have also been departures for two major reasons. First, they rarely produced children. Indeed, none of the 50 workers did so. Second, until recently most died before their parents and in many instances before their grandparents. The average life expectancy of children with Down syndrome in the USA in 1929 was approximately 9 years. Chances are great that such relatively short lives were typical of others with significantly disabling conditions. In 2020, the National Down Syndrome Society estimated that the life expectancy of individuals with Down syndrome to be in the 50 to 60 year range. Chances are great that such enhanced life expectancies are typical of others with significantly disabling conditions. In sum, individuals with significant intellectual disabilities are now participants in the natural order of familial succession, but they cannot survive or thrive without substantial, comprehensive and long term extra support. This presents major problems to families, taxpayers and communities (Brown \& Knollman, 2011).

In 2020, the ages of 44 of the original 50 workers ranged from 34 to 60 . The average age was 53 . This includes the 8 who died. Information about the mortality of 6 workers in unknown. From 1984 to early 2020 , five of the workers who did not die, stop working or leave the Madison area retired from work. One retired at age 42 and died at 46 , one retired at 48 and died at 51 and one retired at 56 and died at 57. Two retired at ages $50 \& 53$. Their mortality information in 2020 was unknown. The average age at retirement was 50. Poor and declining health were the primary reasons. From 1984 to early 2020, four exited the Madison area at ages 33, 35, $41 \& 53$. Information about their mortality status in 2020 was unknown. Three workers stopped coming to work because of 
deteriorating health. One remained on CWS rolls until he died. Another remained on CWS rolls for several months in the hope he would return to work. He did not do so by 2020 . One was involved in a serious accident independent of work. His sibling decided he would not return to CWS.

From 1984 to early 2020, eight workers died. Their ages at death were; $34,38,46,47,51,52,54$ \& 57. One, who had Cornelia deLange Syndrome, choked to death in his residence at age 47 and one, with significant Cerebral Palsy, died for unknown reasons at age 52. Twenty one of the 50 workers had Down syndrome. Ten were female. Six of the eight who died also had Down syndrome. Their average age at death was 46 . The average age of the 13 with Down syndrome who were alive and involved with CWS in 2020 was 55. When the individuals with Down syndrome either died or did not exit CWS by 2020 were combined, their average age was 53. An estimated average life expectancy of 60 for the 19 does not seem unreasonable. Mortality information about the two who left Madison was unknown.

Two of the eight workers who died, did so before their mothers. Three outlived their mothers. It is unknown if the three others who died did or did not do so before their mothers. Three of the eight workers who died did so before their fathers. Two outlived their fathers. It is unknown if the three others who died did or did not do so before their fathers. Two of the eight workers who died did so before both parents. Three of the eight workers who died outlived both parents. It is unknown if the three others who died did or did not do so before their parents.

It was unknown if 10 were dead or alive in 2020. Twenty four workers were alive when their fathers died. That is, they outlived their fathers. Their ages ranged from eight to 57. Their average age when their fathers died was 38 . It could not be determined if ten workers did or did not outlive their fathers. Twenty one workers were alive when their mothers died. That is, they outlived their mothers. Their ages ranged from 11 to 58 . Their average age when their mothers died was 43. It could not be determined if seven workers did or did not outlive their mothers.

\subsection{Guardianship}

Traditionally, parents of children with significant intellectual disabilities assumed they would and should become the legal guardians of their sons/daughters at age 18. Most went through the steps necessary to do so. During the 1990's, some parents and advocates judged that persons with disabilities should be much more involved in important decisions that affected their lives and questioned the necessity and appropriateness of legal guardianship being automatically assigned to parents. They explored and embraced such concepts as self determination, personalized choices and supports, supported decision making, limited guardianship and circles of friends and applied them to the guardianship process. While such alternatives to automatic parent guardianship worked well for some individuals and families, many of the parents of the 50 workers of concern here established more traditional guardianship arrangements. None of the 50 workers was intellectually or otherwise capable of actually functioning as their own legal guardian. If one was considered her/his legal guardian, she/he was assisted by a concerned and informed support team. In 2005 the legal guardian status of the 50 workers was as follows. Thirteen were their own legal guardians; one or more parents were the legal guardians of 35 workers; and, two workers had court appointed guardians. By 2020, 14 of the 50 original workers had died, moved from Madison or retired. CWS has no guardianship information about them. Of the 36 who were still supported by CWS, nine were their own legal guardians, parents remained or became the legal guardians of 12 and siblings remained or became the legal guardians of nine. Six workers had court appointed legal guardians.

\subsection{Social integration}

In the 1970's and 1980's the postschool realities for the workers of concern here were confinement to a sheltered workshop, an activity center or their residences. Some rejected such isolating and otherwise unbearably limiting options. They wanted more ordinary lives. That is, they wanted them to live, work and recreate in integrated society. Why were more ordinary and thus integrated options preferred? First, integration offers more opportunities to function in an increased number of environments. These workers functioned in over 100 integrated work environments as well as in public busses, theatres, parks, streets and lunch and break rooms. Second, they were constantly exposed to the best possible social, behavior, dress, communication and work models. That is, they were constantly exposed to ordinary people doing ordinary things. This allowed them to imitate appropriate actions over long periods of time. Third, they judged that some of the most impor- 
tant advantages of integrated life are opportunities to interact with and develop a range of relationships with coworkers and others without disabilities who are not paid to be with them. Indeed, while the vocational achievements reported above are notable, for most workers they were secondary to the social relationships experienced. Fourth, in the 1970's these and other individuals with significant intellectual disabilities did not go to real schools, busses, theatres, gyms, churches or stores. Indeed, they were rarely seen in public. The workers reported about here helped to change that. Now, more and more individuals with similar disabilities attend real schools, work in real places, interact with thousands of individuals without disabilities, ride public busses, use valuable curb cuts and otherwise show what they can do and what they can overcome to make contributions to society. After centuries of segregation and isolation large numbers of individuals without disabilities are now in their lives. The benefits of functioning in integrated settings were not restricted to the workers. Many coworkers described their work environments as being enhanced, their morale improved and their personal and professional lives more fulfilling because they worked with persons with disabilities.

Mac began working at Madison Fire Station \#1 in 1987. He completed a wide variety of cleaning tasks. Eventually he was able to expand his work to Madison Fire Station \#4. Like most of us, some work days were easier and more productive than others. However, what was always there was the camaraderie that Mac and his coworker fire fighters shared when they were together. In 2012, after working for the Madison Fire Department for over 25 years, he suddenly died. He was given a rare Fire Fighters Memorial Service. This included his parents being presented with an American flag and bagpipes playing at his internment. Words cannot express the comfort, pride and gratitude Mac's family experienced. They realized their son/brother/nephew/cousin/uncle had another family in his life who loved and appreciated him and honored his struggles.

Lena loved many she interacted with while meeting her recycling pick up and shredding responsibilities at Dane County Courthouse, but Judge Meurer was her favorite. At one point her supervisor was faced with an overcrowded building and a lengthy remodeling project. Thus, Lena was scheduled to move to a new location. Her Job Coach explained to Judge Meurer that changes were problematic for Lena. Without hesitation he created a solution. Lena would work in his office and share his space as he would usually be in court. Most mornings at 10:00, Judge Meurer would take a break with Lena and they would chat about their favorite colors, socks, names and foods. Judge Meurer often commented to CWS personnel about the sad lives of some of the people he sees in criminal court every day and what a contrast it was to spend time with Lena. He described their morning times together as the highlights of his day. After the remodeling, she returned to her permanent space. Her friendship with Judge Meurer deepened. She was introduced to his wife and family and engaged in social activities with them outside of work on a regular basis. When Lena's brother was no longer able to fulfill his responsibilities as her legal guardian, Judge Meurer was asked to consider doing so. Just as there was no hesitation to share his office, he immediately said he would be honored to be her guardian.

Toby worked in the WI Bureau of Quality Assurance since 1987 . His early days were replete with challenges. Some days he completed very little work, would run out of the office and would bang his head with his fists, a behavior which eventually resulted in detached retinas in both eyes. Once, while especially agitated he ran out of the office, bumped into a coworker and knocked her to the floor. This resulted in a formal complaint to Human Resources. Fortunately, during these challenging times, he had a champion, his boss Pat. She was able to diffuse the situation with Human Resources, defended him when coworkers questioned his productivity and arranged for inservice training about Autism. She also made sure that Toby was included in all office events and made a point to celebrate all of Toby's positive attributes and interests. She made sure his coworkers knew about his love of farm equipment and his fascination with trains. As relationships developed, his coworkers felt much more comfortable, witnessed fewer elopements and fewer incidents of self abuse. Instead, they experienced more time chatting about tractors, manure spreaders and trains. Pat's relationship extended out of the office. She loved to organize lunches for birthdays and holidays and even took Toby on a train trip to Chicago to the Shedd Aquarium. When she retired she continued to come to his birthday celebrations at work, and they got together when the Baraboo Circus Train rolled into town. They also continued to visit Toby's favorite farm equipment dealer. Twenty nine years later, most of Toby's original coworkers are gone. The newer coworkers are shocked when anyone describes the early days. Toby has had several terrific supervisors, but Pat was his champion. 


\section{Discussion}

From 1981 to 2020 the 50 workers functioned in over 150 integrated work settings for over 570 years, performed hundreds of real work tasks effectively and enjoyed hundreds of social relationships with persons without disabilities who were not paid to interact with them. Not one moved from an integrated to a segregated setting and not one instance of physical or sexual abuse in an integrated setting was reported. Vulnerable individuals are safest when they engage in meaningful activities in places with many peers without disabilities who are not paid to be with them.

The segregationists who opposed allowing opportunities for integrated vocational functioning in the 1970's and 1980's and predicted failure and harm were wrong. Those who oppose integration today are even more wrong because we now have an ever increasing body of evidence that, given authentic assessment and instruction and reasonable long term and personalized extra supports, individuals with significant intellectual disabilities can function successfully and safely in integrated vocational and related environments over long periods of time. The number of environments to which the workers were given access, the quantity and complexity of the tasks they performed, the social relationships they experienced and the personal choices they were allowed to make were enhanced dramatically because they functioned in integrated rather than in segregated settings. If MMSD personnel did not provide authentic vocational assessment and instruction in the 1970's, it is extremely doubtful: that many parents would have advocated for their children to be supported in integrated work settings after school exit; that CWS would have been created; and, that the workers would have had opportunities to function in integrated society for so long.

Tragically, the service delivery models and curricula that have been proven effective preparing students with significant intellectual disabilities for the real world of work at school exit are being used by too few school districts. The results are waste, unemployment, disappointment, frustration, dependence and lives with other descriptors that are less than acceptable. It does not have to be this way. Schools are time limited means to ends. They are not ends. In the USA, students with significant intellectual disabilities typically attend school until around age 22. They are provided specially trained teachers, a wide array of therapies, paraprofessionals, door to door travel services, low ratio instruction, special art, music and physical education, special facilities and individualized curricula. These special services cost substantially more than those offered peers without disabilities. Problematically, they are much more than the monies per capita available to the same persons in postschool service agencies. The results of these well intentioned and expensive services are unacceptable. What can be done to produce better school outcomes?

- Provide increasing kinds and amounts of authentic vocational assessment and instruction as school exit approaches.

- Teach successful functioning in integrated schools and classes, but also in integrated lunch and break rooms, on public busses and trains, in carpools, worksites, parks and in a variety of other integrated settings.

- Teach students to do as much as possible for themselves and to function as well as possible under natural supervisory conditions. The unnecessary use of one to one and other low ratio instructional arrangements during school careers make it extremely difficult for individuals with disabilities to function in real work and related settings under natural supervisory conditions and in accordance with financially viable Job Coach to worker ratios at school exit.

- Teach as many students without disabilities as possible, the coworkers, supervisors and employers of the future, to provide meaningful natural support.

- Teach students to be nice to others and to work hard. If you are nice and work hard, it is amazing how coworkers without disabilities, supervisors and employers will extend themselves so you can be successful.

- Take responsibility for, plan for, fight for and otherwise do all that is feasible to produce integrated outcomes.

An important common denominator of persons with significant intellectual disabilities is their need for long term extra support. This was understood by the courageous and insightful Dane County officials who generated and maintained it. If not for their values, priorities and commitments, the workers, and many others, would have spent their lives in segregated settings or at home. In 2020, 14 agencies in Dane County, WI provided services to approximately 1,100 workers with a wide variety of disabilities who required long term extra support in integrated work environments. 
Personnel to worker ratios are causally and inversely related to costs: the higher the ratio, the lower the costs; the lower the ratio, the higher the costs. This economic reality is a major problem for all persons with disabilities who wish to function in integrated vocational settings but need extra support. The more you cost, the higher are the probabilities that you will be sent to segregated settings, placed on waiting lists and/or confined to your residence. In addition, as the number of persons with disabilities who need long term taxpayer support for vocational services increases, so does the urge to revert to less than acceptable sheltered workshops and activity centers. For example, persons with significant personal care needs who use wheelchairs are often monitored using one to eight or more ratios in segregated settings. If the same persons were given opportunities to function with reasonable assistance in integrated work settings, such high ratios would be inconceivable.

CWS operates with a one to 4.6 ratio because it serves persons who need lifetime supports. Indeed, most of the workers are aging sooner than chronological age peers without disabilities. Dementia, mental illness and loss of physical functioning are increasingly problematic.

Nevertheless, they express a reasonable range of functioning levels, behavioral challenges, personal care repertoires, etc. Few agencies can survive financially or otherwise if they only serve workers with complicated personal care needs or only those who express serious behavioral challenges. Balanced and heterogeneous populations allow more persons with disabilities to function in integrated settings at reasonable costs.

If you are alive and have significant disabilities, you must be somewhere. Where should you be? You must be with someone. Who should you be with? You must be doing something. What should you be doing? You should be in respected environments with individuals without disabilities doing what they do because an integrated life is inherently better than one that is segregated. We must do all that is reasonable to prevent anyone from experiencing lives that are segregated, nonproductive, sterile, unnecessarily dependent and costly. Conversely, we must do all that is reasonable to prepare and arrange for all citizens to live, work and recreate enjoyably and productively in safe, stimulating and diverse integrated society; $i$ $\mathrm{e}$, to live lives that are as ordinary as possible.

Finally, in 2020 the population of the USA was approximately 320 million. The lowest intellectually functioning $1 \%$ of 320 million was 3.2 million. Do the lives of those in this sample of 50 represent those of the 320 million? No, but if we wanted them to they could. Hundreds of employers and supervisors in the Madison area opened their doors, hearts, values and pocketbooks and afforded these 50 deserving individuals a wonderful array of opportunities. Are they representative of the many millions of employers and supervisors in the USA? We say yes, so let's give them the opportunities necessary to demonstrate they are. Thousands of coworkers without disabilities sat next to, touched, helped, bumped into, learned to communicate with, worked with, shared restrooms with, laughed with, lunched with the lowest intellectually functioning $1 \%$ of the adults in their community. Are they representative of the millions of coworkers without disabilities around the world? We say yes, so let's go and get to know them. Finally, there is one more important lesson that emerges from the data presented. Each of these workers was more capable than almost everyone who knew them when they were young presumed. We now realize they can laugh, love, cry, produce real work, help others, take pride from their achievements and otherwise surprise and inform us with their previously hidden abilities. All they needed was for the village to create access to the arena and provide the critical extra support.

\section{Conflict of interest}

None to report.

\section{References}

Blackorby, J. \& Wagner, M. (1996). Longitudinal postschool outcomes of youth with disabilities: findings from the national longitudinal transition study. Exceptional Children, 62, 399413.

Brown, L., Kessler, K., \& Toson, A. (2016). An integrated work skill analysis strategy for workers with significant intellectual disabilities. Journal of Vocational Rehabilitation, 44(1), 73-83. https://doi.org/10.3233/JVR-150781

Brown, L. \& Kessler, K. (2014). Generating integrated work sites for individuals with significant intellectual disabilities. Journal of Vocational Rehabilitation, 40, 85-97. https://doi.org/10.3233/JVR-140675

Brown, L. \& Knollman, G. (2011). Social justice and individuals with significant intellectual disabilities. TASH Connections, 37(2), 7-12.

Brown, L., Nietupski, J. \& Hamre Nietupski, S. (1976). The criterion of ultimate functioning and public school services for severely handicapped students. Hey, Don't Forget About Me: Education's Investment in the Severely, Profoundly and 
Multiply Handicapped, 2-15. Reston, Virginia: Council for Exceptional Children.

Brown, L., Nisbet, J., Ford, A., Sweet, M., Shiraga, B., York, J. \& Loomis, R. (1983). The critical need for nonschool instruction in educational programs for severely handicapped students. The Journal of the Association for Persons with Severe Handicaps, 8(3), 71-77.

Brown, L., Shiraga, B., \& Kessler, K. (2006). The quest for ordinary lives: The integrated post-school vocational functioning of 50 workers with significant disabilities. Research and Practice for Persons with Severe Disabilities, 31, 93-121.

Certo, N., Pumpian, I., Fisher, D., Storey, K., \& Smalley, K. (1997). Focusing on the point of transition. Education and Treatment of Children, 20, 68-84.

Certo, N. J., Sax, C., Pumpian, I., Mautz, D., Smalley, K., Wade, H. A. \& Noyes, D. (2002). Transition service integration model: Ensuring that the last day of school is no different than the day after. In C. Sax \& C. A. Thoma (Eds.) Transition assessment: Wise practices for quality lives (pp. 119-131). Brookes Publishing Company.

Cutler, S., \& Schmid, S. (2020). The Community Work Services Cumulative Worker Record. Retrieved from www.cwsmadison.com.
Horvath, J., Rose, A., \& Stapleton, D. C. (2003). Trends in outcomes for young people with disabilities: Have policies aimed at social integration and economic independence been effective? Rehabilitation Research and Training Center for Economic Research on Employment Policy for Persons with Disabilities. Cornell Center for Policy Research: Ithaca, NY.

Luecking, R., \& Certo, N. (2003). Integrating service systems at the point of transition for youth with significant support needs: a model that works. American Rehabilitation, 27(1), 2-9.

Murphy, S. \& Rogan, P. (1995). Closing the shop: Conversion from sheltered to integrated work. Brookes Publishing Company.

PCESE. (2002). A new era: revitalizing Special Education for children and their families. The President's Commission on Excellence in Special Education. Washington, D.C.

PCID. (2004). A roadmap to personal and economic freedom for people with intellectual disabilities in the 21st century. The President's Committee for People with Intellectual Disabilities. Washington, D.C.

Wehman, P., \& Kregel, J. (1998). More than a job: Securing satisfying careers for people with disabilities. Brookes Publishing Company. 